\title{
HYPEROSMOLARITY NORMALISES SERUM-INDUCED CHANGES TO CHONDROCYTE PROPERTIES IN A MODEL OF CARTILAGE INJURY
}

\author{
A. Karim and A.C. Hall* \\ Centre for Integrative Physiology, Deanery of Biomedical Sciences, University of Edinburgh, Edinburgh, \\ Scotland, UK
}

\begin{abstract}
Partial-thickness cartilage injuries do not heal effectively, potentially leading to degeneration as occurs in posttraumatic osteoarthritis (PTOA). The role of chondrocytes could be crucial in determining the nature of the repair; however, their response to this injury is poorly understood. We have utilised an in vitro bovine osteochondral partialthickness scalpel injury model and determined chondrocyte properties at and distant from the injury in the presence/ absence of (a) serum-free DMEM (340 mOsm), (b) synovial fluid DMEM (SF-DMEM), (c) foetal calf serum DMEM (FCS-DMEM), (d) hyperosmolar serum-free DMEM (600 mOsm), or (e) hyperosmolar FCS-DMEM for up to two weeks. Chondrocytes were fluorescentlylabelled with 5-chloromethylfluorescein-diacetate (CMFDA)/propidium iodide (PI) for live/dead cells and imaged using confocal microscopy. Quantitative data were obtained on chondrocyte properties (cell volume, clusters, morphology) at and distant from the injury. In serum-free DMEM, chondrocyte morphology at the injury remained unaffected throughout culture. However, with SF-DMEM or FCS-DMEM the chondrocytes displayed an increase in volume $(p<0.0001)$, cluster formation (FCS; $p<0.01)$ and abnormal morphology $(p<0.001)$ compared to serum-free DMEM. Cluster formation and shape changes during FCS-DMEM culture were more pronounced than with SF-DMEM. SF-DMEM or FCS-DMEM stimulated these changes to chondrocytes at the injury with only small effects on distant cells. Hyperosmolarity inhibited the morphological and volume changes to chondrocytes induced by FCS-DMEM $(p<0.001)$ and the injured cartilage had the appearance of that in serum-free DMEM. Raised osmolarity may therefore have benefit in preserving the morphological phenotype of chondrocytes at the site of injury, and thus promote more effective integrative repair in partial-thickness cartilage injury.
\end{abstract}

Keywords: Cartilage, chondrocyte, injury, clustering, morphology, serum, synovial fluid, hyper-osmolarity, culture.

*Address for correspondence:

Dr. Andrew C. Hall

Centre for Integrative Physiology

Deanery of Biomedical Sciences

Hugh Robson Building, George Square

Edinburgh EH8 9XD, Scotland, UK

Telephone Number: +44 (0)1316503263

FAX Number: +44 (0)1316502872

E-mail: a.hall@ed.ac.uk

\section{Introduction}

It is well established that the ability of adult articular cartilage to repair is critically dependent on whether or not an injury penetrates the full thickness to the calcified cartilage of subchondral bone (Hunziker, 2002). Injuries which breach the bone expose the vascular system and bone marrow to the defect void, and can stimulate an intrinsic cartilage 'repair' response (Steadman et al., 2001). This involves fibrin clot formation, vascular invasion and recruitment of mesenchymal stem cells into the defect, resulting in chondrogenesis at the wound site. However, the repair is fibro-cartilaginous (i.e. scar tissue) in nature, and there is a change in the morphology of chondrocytes from their classical 'normal' elliptical/rounded shape to an abnormal morphology more characteristic of a dedifferentiated 'fibroblastic' phenotype (Hunziker, 1999). The extracellular matrix (ECM) formed generally has a higher ratio of collagen type I to II, less proteoglycan (aggrecan) and inferior biomechanical properties and little morphological or structural similarity to hyaline cartilage, and is therefore vulnerable to degeneration (Knutsen et al., 2004).

In contrast, partial-thickness injuries cannot be accessed by blood-borne cells and do not heal. Their appearance several months later is similar to that at the time of injury and they appear to be inert unlike similar defects in immature articular cartilage which show evidence of recovery (Wei et al., 1997; Namba et al., 1998). Recent work suggests that the response of cartilage to these injuries is not, in fact, negligible and that there are potentially important cellular changes occurring at the injury site. For example, following culture of human cartilage subjected to partial-depth injury, some changes to chondrocytes have been described (Lyman et al., 2012). In addition, Seol et al. (2012) have reported the presence of chondrogenic progenitor cells (CPCs), possibly attracted by 'alarmins' (or damage associated molecular pattern molecules) released by necrotic chondrocyte death. It has been suggested that CPCs actively migrate to the site of injury following short-term enzymatic treatment of cartilage (Seol et al., 2014). Studies of the cellular response to these injuries are therefore of considerable interest as they might clarify why effective repair does not occur.

Clearly, partial-thickness injuries will damage components of the ECM leading to complex changes including disruption of the collagen type II structure and progressive leaching/loss of matrix proteoglycans (Patwari et al., 2003). The damage to collagen architecture will reduce the restraining force on the partially-inflated aggrecan molecules (Maroudas, 1976), resulting in 
further imbibition of water/tissue swelling at the injury (Maroudas and Venn, 1977). The osmolarity of cartilage interstitial fluid, and thus that to which the chondrocytes are normally exposed, is higher than that of normal tissue culture solutions (approx. 350-450 mOsm depending on cartilage zone, compared to approx. 280-340 mOsm; Urban et al., 1993). Thus, cartilage injury would be expected to cause interstitial osmolarity at/near the injury to fall towards that of the extracellular solution. Chondrocyte volume is directly influenced by osmolarity (Bush and Hall, 2001a) and this has a powerful effect on cellular responses, including ECM metabolism (Urban et al., 1993). For example, alterations to the osmotic environment regulate the expression of the chondrogenic transcription factor SOX9 (de Crombrugghe et al., 2000) that is essential for the expression of many genes encoding ECM proteins, including collagen type II (Col2a1) and aggrecan (Tew et al., 2009). Matrix damage may also promote access of growth factors and other agents in the extracellular solution (i.e. serum or synovial fluid in culture medium) and these may stimulate chondrocyte ECM metabolism and/or mitogenic activity (Trippel, 1995; Fortier et al., 2002; Cucchiarini et al., 2005; Byun et al., 2013).

Protecting chondrocytes against the deleterious effects of swelling through reduced extracellular osmolarity and excessive growth factor stimulation might provide an optimal environment to direct chondrocytes to produce an appropriate ECM to repair the cartilage from partialthickness injury. While there have been extensive studies on the response of cartilage to these injuries (Hunziker, 1999; Hunziker, 2002; Redman et al., 2004), there has been less attention paid to the effects of medium osmolarity and serum/growth factors (Hunziker, 2001) which may influence chondrocyte properties at the site of injury. Raising osmolarity reduces chondrocyte death following impact injury (Bush et al., 2005), scalpel cutting (Amin et al., 2008; Amin et al., 2009) and cartilage drilling (Farhan-Alanie and Hall, 2014). It has also been shown that repair of rat cartilage in vivo was more effective following partial-thickness injury performed in the presence of a hyper-osmotic (600 mOsm) irrigation solution compared to normal saline (approx. $280 \mathrm{mOsm}$ ) (Eltawil et al., 2015).

As a first step towards clarifying the response of chondrocytes to cartilage injury, we have performed reproducible partial-thickness injury on bovine osteochondral explants. These have then been cultured in the presence or absence of synovial fluid (SF) or foetal calf serum (FCS) in tissue culture medium of normal (340 $\mathrm{mOsm})$ or raised osmolarity $(600 \mathrm{mOsm})$. Using confocal scanning laser microscopy (CLSM) and imaging methods, we have then quantified the response (viability, volume, morphology, clustering) of fluorescently-labelled in situ chondrocytes at and distant from the injury. Our results demonstrated that there are profound changes to chondrocyte properties at the site of injury which can be suppressed by removal of FCS or raised osmolarity.

\section{Materials and Methods}

\section{Biochemicals and solutions}

Biochemicals were from Invitrogen Ltd. (Paisley, UK) or Sigma-Aldrich (Poole, UK) unless stated otherwise. Standard culture medium was Dulbecco's Modified Eagle's Medium (serum-free DMEM; $340 \mathrm{mOsm}, 37^{\circ} \mathrm{C}$, $5 \% \mathrm{CO}_{2}, \mathrm{pH} 7.4$ ) containing penicillin/streptomycin (100 U/mL and $100 \mu \mathrm{g} / \mathrm{mL}$, respectively). The viability probe, 5-chloromethylfluorescein diacetate (CMFDA) and the nucleic acid dye propidium iodide (PI) were prepared as described (Amin et al., 2008). Formaldehyde solution ( $4 \% \mathrm{v} / \mathrm{v}$ in saline, $\mathrm{pH} 7.3$ ) was from Fisher Scientific (Loughborough, UK). For some experiments, serum-free DMEM was modified by addition of $10 \%$ bovine synovial fluid (SF-DMEM; $340 \mathrm{mOsm}$ ) or $10 \%$ foetal calf serum (FCS-DMEM; $340 \mathrm{mOsm}$ ). SF was taken aseptically from fresh (within $24 \mathrm{~h}$ of slaughter) healthy bovine metacarpophalangeal joints, centrifuged (Hettich Zentrifugen $32 \mathrm{R}, 21^{\circ} \mathrm{C}, 1157 \times \mathrm{g}, 10 \mathrm{~min}$ ) and the supernatant stored $\left(-20^{\circ} \mathrm{C}\right)$ until required. When required, sucrose was added for hyperosmolarity experiments, with osteochondral explants exposed to either (a) normal saline (340 mOsm) or (b) hyperosmolar saline $(600 \mathrm{mOsm}$; Amin et al., 2010) $5 \mathrm{~min}$ before and $5 \mathrm{~min}$ after the application of injury to allow chondrocytes to respond to altered osmotic environment and then cultured under four conditions: (a) serum-free DMEM (340 mOsm), (b) FCS-DMEM (340 mOsm), (c) hyperosmolar serum-free DMEM (600 mOsm) or (d) hyperosmolar FCS-DMEM (600 mOsm).

\section{Cartilage preparation, application of scalpel injury and fluorescent labelling}

Metacarpophalangeal joints of 3 year-old cows were prepared (Amin et al., 2008) and full-depth osteochondral explants trimmed to approx. $5 \times 3 \times 1 \mathrm{~mm}$. The articular surface was then injured by a single pass of a fresh No. 11 blade in a 'push-through mode' down the long axis of the explant (Amin et al., 2009). The injury was always produced in the same direction, avoiding additional movements of the scalpel, cutting carefully without penetrating the bone. The typical depth of the injury was approx. $50 \mu \mathrm{m}$, whereas the thickness of the explant was approx. $800 \mu \mathrm{m}$. The explants were cultured in experimental media for up to $14 \mathrm{~d}$, with media changed on alternate days. At appropriate time points and during the last hour of culture, explants were incubated with CMFDA and PI (12.5 and $5 \mu \mathrm{M}$, respectively) to label living or dead cells, respectively. Explants were then washed in phosphate-buffered saline (PBS), fixed in $4 \%$ formaldehyde (30 min) and mounted for imaging (Amin et al., 2008).

\section{Confocal laser scanning microscopy (CLSM)}

A Zeiss Axioskop LSM510 upright CLSM (Zeiss (UK), Welwyn Garden City, UK) was used with $\mathrm{E}_{\mathrm{x}}$ of $488 \mathrm{~nm}$ and $543 \mathrm{~nm}$ for the fluorophores CMFDA and PI, respectively. Emission light was passed through a band-pass filter $(505-530 \mathrm{~nm})$ and long-pass filters $(650 \mathrm{~nm})$, respectively. Fluorescently-labelled in situ chondrocytes were imaged 
in the axial plane at, and $\sim 400 \mu \mathrm{m}$ distant, from the injury. Chondrocyte viability was assessed using a low power objective ( $\times 10$ dry, NA = 0.3) (Amin et al., 2009), and injury width and chondrocyte morphology studied using a high power $(\times 40, \mathrm{NA}=0.8)$ dipping water $(\mathrm{DW})$ lens. The same region of interest (ROI) was applied for all measurements of chondrocyte viability/morphology. The $z$ stack images comprising single confocal sections typically to a depth of $50 \mu \mathrm{m}$ for high and $100 \mu \mathrm{m}$ for low power objective magnification measurements were collected at intervals of $1 \mu \mathrm{m}$ and $10 \mu \mathrm{m}$, respectively, and frame size of $1024 \times 1024$ pixels. Optical sections were converted into three-dimensional (3D) reconstructions using Volocity ${ }^{\mathrm{TM}}$ (Improvision, Coventry, UK) software (Amin et al., 2008).

\section{Quantification of live and dead chondrocytes}

Low-power axial confocal images were analysed for live/ dead cell counting using the green/red staining pattern, respectively, with an automated counting protocol on Volocity ${ }^{\mathrm{TM}}$ (Amin et al., 2009). A 3D ROI was created to encompass the injured site with dimensions of $x, y, z$ of 400 , 913 and $100 \mu \mathrm{m}$, respectively. Cell death distant from the injury was determined by applying ROIs with dimensions of $x, y, z$ of 200,913 and $100 \mu \mathrm{m}$, respectively, on both sides of the injury and the average calculated. Therefore, the area was $\sim 200 \mu \mathrm{m}$ distant from the injury on either side on the same image. The counting protocol identified objects (individual cells) as green (live) or red (dead) on the basis of percentage threshold intensity in the two channels. The mean number of live/dead cells in each ROI was calculated and \% PI-labelled cells (i.e. percentage cell death, PCD) determined as $100 \times$ (number of dead cells/ number of dead and live cells $) \%$. The high power $(\times 40$ DW) axial confocal images were analysed by Volocity ${ }^{\mathrm{TM}}$ software to determine the width of the injury by drawing two straight lines on either side of the injury enclosing the area containing no cells and measuring the distance between the lines. Chondrocyte volume/morphology were determined by analysing high power images 'at' and 'distant from' the injury within the specified ROI with the dimensions of $x, y, z$ of 228,228 and $50 \mu \mathrm{m}$, respectively, applied at the injured site and approximately $400 \mu \mathrm{m}$ distant from the injury on separate images.

\section{Volume and morphological analysis of in situ chondrocytes}

Quantitative data regarding volume and 3D morphological analysis of in situ chondrocytes were obtained from the CLSM ( $\times 40$ DW objective) images using a measurement protocol on Volocity ${ }^{\mathrm{TM}}$. In order to determine chondrocyte volume, it was necessary to identify the cell edge and this was done using a threshold segmentation method, and volume determined individually. Volume was calibrated by determining the baseline threshold intensity of fluorescent latex beads (Fluoresbrite ${ }^{\mathrm{TM}}$, Polyscience Inc., Warrington, UK; Bush and Hall, 2001a).

Chondrocytes in superficial zones of bovine cartilage typically exist in pairs (Sasazaki et al., 2008) and thus we defined a cluster as consisting of $\geq 3$ chondrocytes within the lacunar space. Chondrocytes lying at the edges of images were not included in the analysis. The number of cells in a cluster was counted manually because it was impossible to segment individual cells as the cell membranes were frequently touching. The total number of chondrocytes in CLSM projections in a fixed ROI was counted, and the percentage of cells involved in forming clusters calculated as: $\%$ cells in a cluster $=100 \times($ number of cells forming clusters/total number of cells) $\%$. For measurements of cell morphology, the cell body of individual chondrocytes was measured along the longest $(y)$ axis of the cell in $3 \mathrm{D}$ using Volocity ${ }^{\mathrm{TM}}$. Chondrocytes which were elliptical/ spheroidal with no cytoplasmic processes were considered morphologically 'normal'. However, cells with elongated/ non-spheroidal shapes, frequently including cytoplasmic processes, were considered morphologically 'abnormal' (Bush and Hall, 2003). These chondrocytes had a variety of characteristics based on (a) shape of cells, (b) presence or absence of cytoplasmic processes, and (c) presence of single or multiple processes (examples illustrated in Results). The percentage of abnormal chondrocytes was calculated as: $\%$ abnormal chondrocytes $=100 \times($ number of abnormal cells/total number of normal and abnormal cells) $\%$.

\section{Data presentation and analysis of results}

Data were presented as mean \pm standard error of the mean (SEM) with $N$ representing the number of different animals, $n$ the number of explants and $n^{\prime}$ the number of cells for each experimental condition. Statistical tests and graphs were generated using Graph Pad Prism 6 (GraphPad Inc., La Jolla, CA, USA). Unpaired, two-tailed, Student's $t$-tests were used to compare means within groups. Oneway analysis of variance (one-way ANOVA) was used to compare data between groups. A significant difference was accepted when $p<0.05$. ' $a$ ' was used to show significant difference using Student's $t$-tests within groups, ' $b$ ' was used to show significant difference using ANOVA between the groups compared, and ' $C$ ' was used to indicate a statistically significant difference using the Student's $t$-test between data obtained at and distant from the injury and between standard and hyperosmolar culture conditions. Single, double and triple symbols showed the level of significance for $p<0.05,0.01$ and 0.001 , respectively.

\section{Results}

\section{Response of cartilage and chondrocytes following culture with serum-free DMEM, synovial fluid- DMEM (SF-DMEM) or foetal calf serum-DMEM (FCS-DMEM)}

Fig. 1A(a-j) shows the overall response of cartilage and in situ chondrocytes at the site of scalpel injury following culture under different conditions. After $14 \mathrm{~d}$ in serum-free DMEM, the rounded morphology of chondrocytes did not change (Fig. 1A(a-d)). However, by day 14 in the presence of synovial fluid (SF-DMEM; Fig. 1A(e-g)) or foetal calf serum (FCS-DMEM; Fig. 1A(h-j)), abnormal chondrocyte morphology was evident with cells developing long cytoplasmic processes in the direction of the scalpel injury.

In serum-free DMEM, injury width increased significantly over the $14 \mathrm{~d}$ culture period $(p<0.05$ by 
A

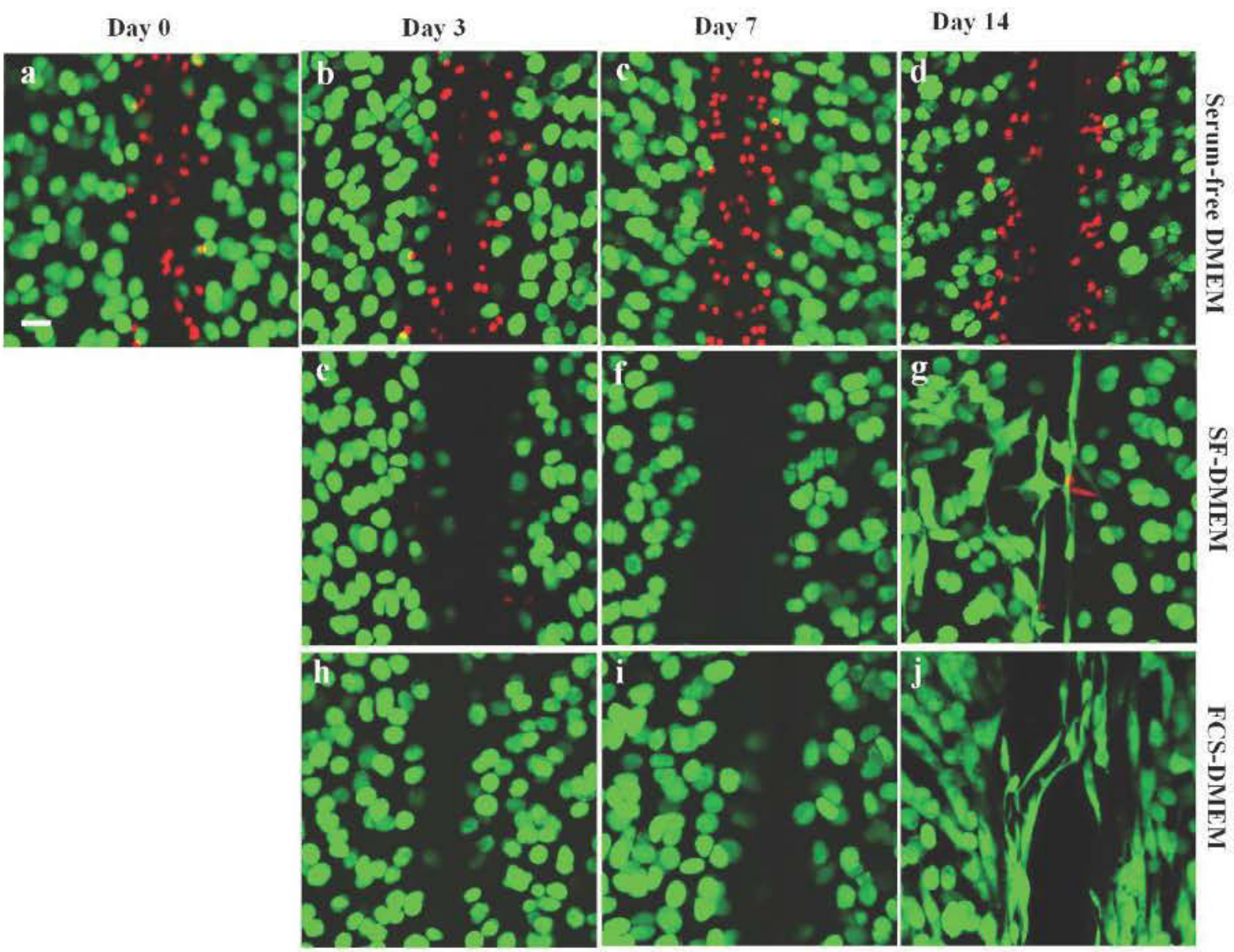

B

C
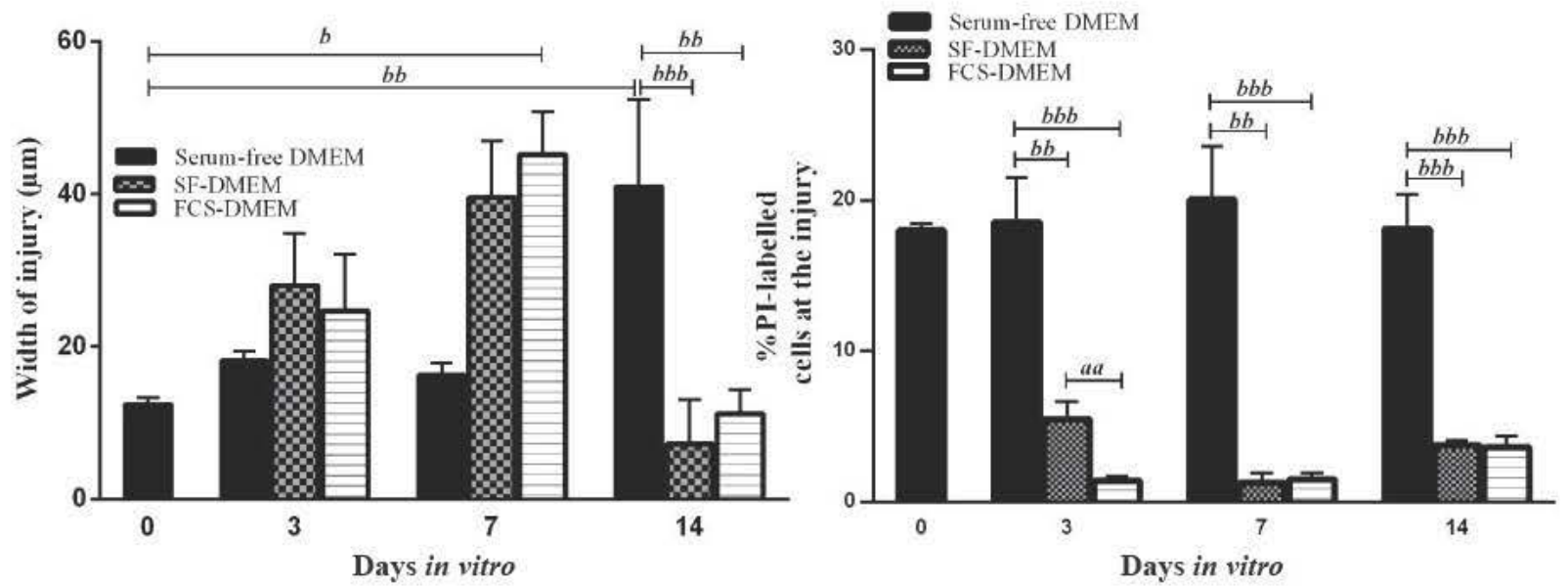

Fig. 1. Response of chondrocytes at the injury following culture under various conditions. (A)Axial CLSM high power (×40 DW) magnification reconstructed images of CMFDA (green fluorescence) and PI-labelled (red fluorescence) chondrocytes (live/dead, respectively) in injured explants at (a) day $0,(\mathbf{b}, \mathbf{e}, \mathbf{h})$ day $3,(\mathbf{c}, \mathbf{f}, \mathbf{i})$ day 7 and $(\mathbf{d}, \mathbf{g}, \mathbf{j})$ day 14 cultured in serum-free DMEM, SF-DMEM and FCS-DMEM, respectively. Scale bar for all panels $=25 \mu \mathrm{m}$. (B) Width of injury at various time points under various culture conditions. Data were from $[N(n)]=[9(43)]$. (C) The $\%$ PI-labelled cells at the injury under the three culture conditions. Data were from $[N(n)=18(52)]$. $a$ indicates a significant difference between two culture media at one time point and $b$ indicates significant difference between various culture conditions at different time points. Single, double and triple symbols indicate the level of significance for $p<0.05,0.01$ and 0.001 , respectively. 
A

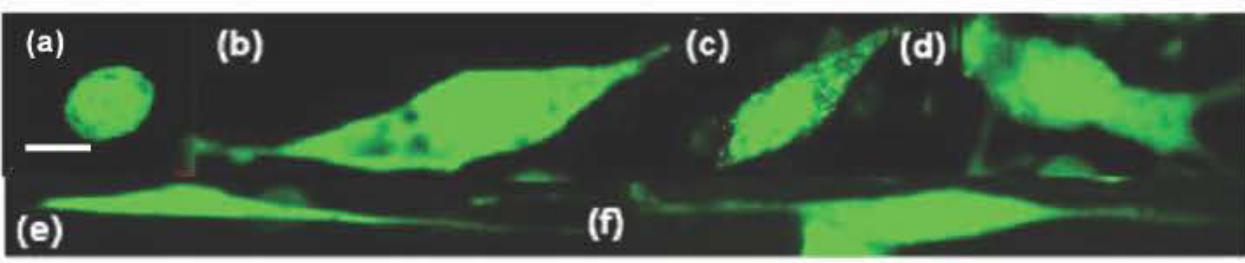

$\%$ of cells with processes

At the injury

B

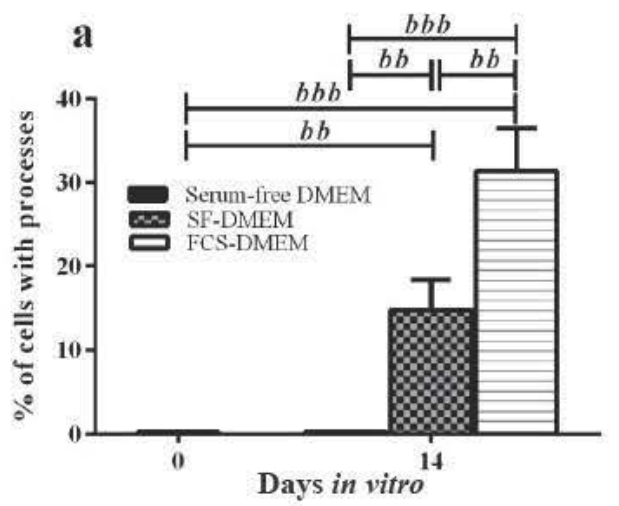

Distant from the injury

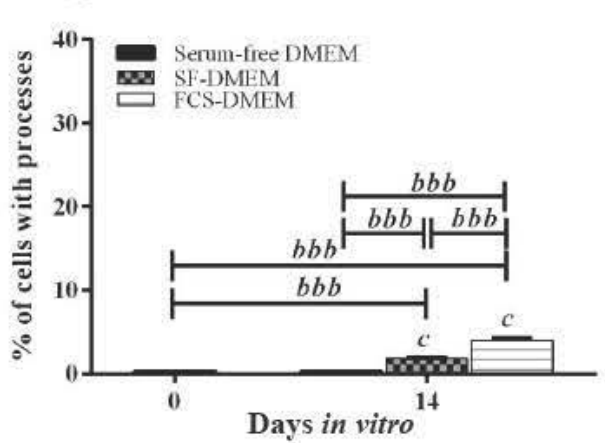

Volume of cells

C

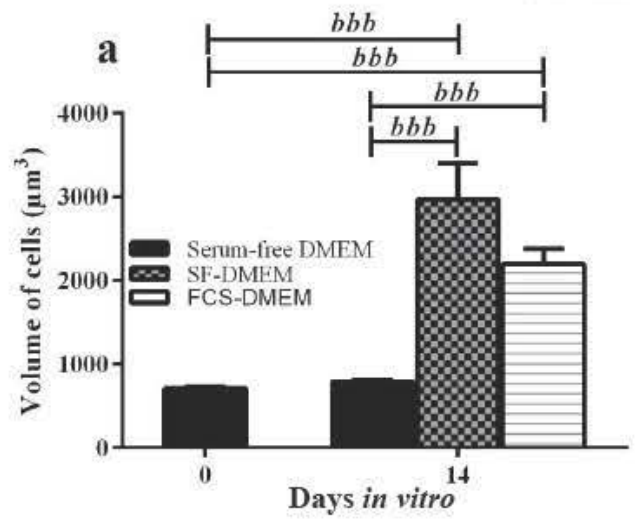

b

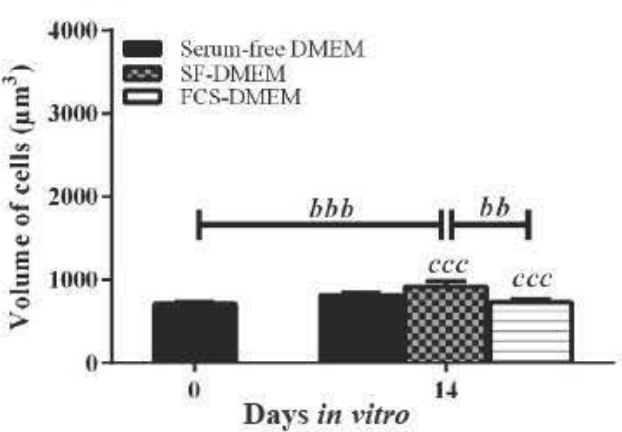

Length of cell body

D

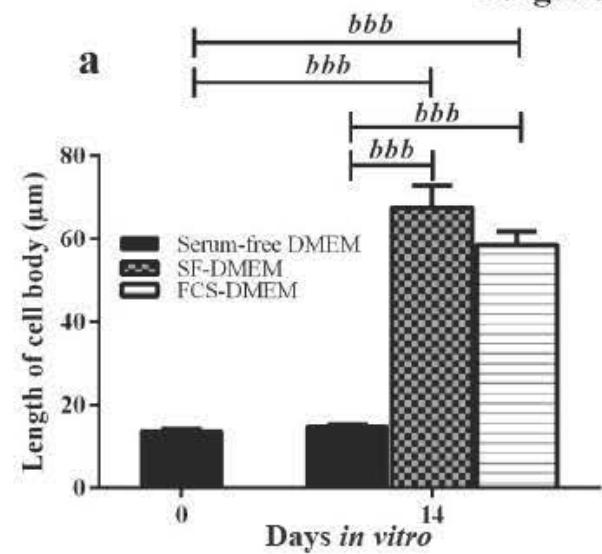

b

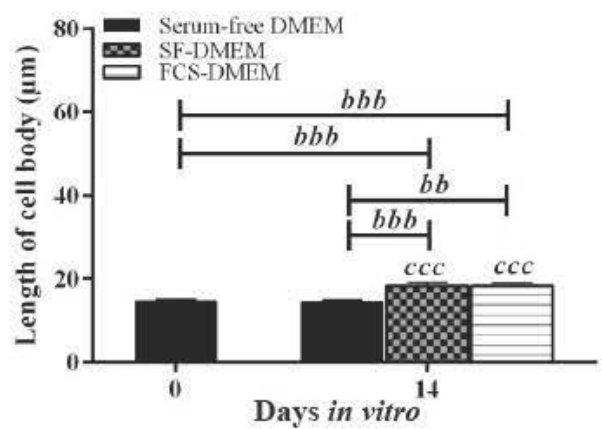

Fig. 2. Examples and characteristics of the heterogeneous nature of chondrocyte morphology. (A) Examples of abnormal chondrocytes in response to injury in the presence of serum-free DMEM, SF-DMEM and FCS-DMEM at day 14 of culture. The varieties of chondrocytes observed were (a) relatively normal with spheroidal shape and (b) abnormal chondrocytes with flattened, elongated cell body. Abnormal chondrocytes had elongated body and single/multiple cytoplasmic processes emanating from the cell body (c, $\mathbf{d}, \mathbf{e}$ and $\mathbf{f})$. Images were obtained by CLSM ( $\times 40$ DW objective) and the scale bar for all panels $=10 \mu \mathrm{m}$. Graphs show pooled data for (B) $\%$ abnormal cells (a) at and (b) distant from the injury, (C) average volume $\left(\mu \mathrm{m}^{3}\right)$ of cells (a) at and (b) distant from the injury, and (D) average length $(\mu \mathrm{m})$ of cell bodies (a) at and (b) distant from the injury. Data were from $[N(n)]=[18(23)] . b$ indicates a significant difference between various culture conditions at two time points, and $c$ indicates a significant difference between at and distant from the injury. Single, double and triple symbols showed the level of significance for $p<0.05,0.01$ and 0.001 , respectively. 
ANOVA; Fig. 1B). For explants in SF-DMEM and FCSDMEM, a significant increase was also observed but only up to day 7 , since between days 7 and 14 , the width of the injury decreased significantly for both conditions $(p<0.01$ and $p<0.001$, respectively, by Student's $t$-tests). The width was also less compared to that of explants cultured in serum-free DMEM (Fig. 1B; $p<0.001$ and $p<0.01$, respectively). The images (Fig. $1 \mathbf{A}(\mathbf{g}, \mathbf{j}))$ indicated that this was most likely due to the presence of abnormal chondrocytes in the injury cleft resulting from the presence of SF or FCS in the culture media.

Images of fluorescently-labelled cells at the injury demonstrated a significant decrease in PI-labelled material in the presence of SF-DMEM and FCS-DMEM even at day 3 (Fig. 1A(e-g), (h-j) and Fig. 1C). However, there was no such effect in serum-free DMEM with red fluorescent PI-labelling clearly persisting (Fig. 1A(a-d) and Fig. 1C) with no significant change ( $p>0.05$ by ANOVA) over $14 \mathrm{~d}$ of culture. In marked contrast, the \% PI-labelled cells at the injury in the presence of SF-DMEM and FCS-DMEM was significantly less throughout the culture period compared to serum-free DMEM (Fig. 1C; $p<0.01$ by ANOVA). Thus, in the presence of SF-DMEM and FCS-DMEM, PI-labelling decreased by approx. 80-90 \% compared to serum-free DMEM throughout the culture period. In addition, at day 3 there was significantly more PI-labelling in explants cultured in SF-DMEM compared to FCS-DMEM (Fig. 1C; $p<0.01$ by Student's $t$-test), suggesting that at this point FCS was more effective at removing PI-labelled material.

\section{Morphological characteristics of chondrocytes at, or distant from, the injury following culture with SF- DMEM or FCS-DMEM}

Chondrocyte morphology at the injury was relatively 'normal' (elliptical/spheroidal in shape with no cytoplasmic processes) at days 0,3 and 7 under all culture conditions (Fig. 1A). However, by day 14, chondrocytes displayed marked shape changes in the presence of SF-DMEM and FCS-DMEM, which were characterised by production of cytoplasmic processes, cell enlargement and elongation of the cell body (examples in Fig. 2A(a-f)). In contrast, cells in the injured region of cartilage cultured in serum-free DMEM still exhibited normal morphology. In order to compare these changes with cells distant from the injury, properties of chondrocytes (abnormal morphology, cell volume, length of cell body) were quantified as described (see Materials and Methods) with pooled data presented in Fig. 2B-D. The morphological characteristics of chondrocytes at day 14 were compared between relatively 'normal' and 'abnormal' chondrocytes following culture in serum-free DMEM and SF- or FCS-DMEM respectively.

After $14 \mathrm{~d}$ of culture, $15 \pm 3 \%$ cells in SF-DMEM and $31 \pm 5 \%$ cells in FCS-DMEM showed significant morphological changes compared to chondrocytes at the injury in serum-free DMEM (Fig. 2B(a); $p=0.0013$ and $p<0.0001$, respectively) and chondrocytes at day 0 ( $p<0.01$ and $p<0.001$, respectively) which were all morphologically 'normal'. Distant from the injury after $14 \mathrm{~d}$, chondrocytes with abnormal morphology were only very occasionally observed (Fig. 2B(b)). However, there were more chondrocytes with processes during culture with FCS-DMEM compared to SF-DMEM (Fig. 2B(a); $p<0.01$ ), suggesting a more potent effect of FCS. The percentage of abnormal cells was significantly greater as compared to distant from the injury in explants cultured with SF-DMEM or FCS-DMEM (Fig. 2B(b); $p<0.05$ ). Additionally, in the presence of serum-free DMEM, chondrocyte morphology remained unaffected at, or distant from, the injury (Fig. 2B(a,b)).

There was an overall three-fold increase in the volume of morphologically-abnormal chondrocytes at the injury in the presence of SF-DMEM or FCS-DMEM compared to serum-free DMEM (Fig. 2C(a)). The average volume of individual abnormal chondrocytes by day 14 was significantly higher in SF-DMEM and FCS-DMEM compared to serum-free DMEM and also in comparison to the volume of chondrocytes at day 0 (Fig. $2 \mathbf{C}(\mathbf{a})$; $p<0.0001)$. However, distant from the injury in the presence of SF-DMEM or FCS-DMEM, the volume of chondrocytes was significantly lower than those at the injury (Fig. $2 \mathbf{C}(\mathbf{b}) ; p<0.0001)$. In the presence of serum-free DMEM, the volume of chondrocytes remained unaffected at or distant from the injury.

The length of the cell body of individual abnormal cells at the injury increased by approximately 4-fold in the presence of SF-DMEM and FCS-DMEM compared to serum-free DMEM and also day 0 (Fig. 2D(a); $p<0.0001)$. The length of cell bodies at the injury was significantly greater compared to those distant from the injury following culture in SF-DMEM or FCS-DMEM (Fig. 2D(b); $p<0.0001$ ). In serum-free DMEM, the length of cell bodies remained unaffected at or distant from the injury, suggesting no morphological abnormalities. These results suggested that by day 14 , chondrocyte volume/ morphology remained unaffected in serum-free DMEM but displayed marked changes in the presence of SF-DMEM or FCS-DMEM.

Measurements of individual chondrocyte volumes were performed at an earlier time point, i.e. day 3 , because later during the culture chondrocytes formed clusters and it was difficult to accurately segment the cells. After $7 \mathrm{~d}$ in culture, measurements for clusters were performed since with further culture, due to the complex nature of the changes occurring, identification of individual cells in a cluster was unclear. The morphology of chondrocytes was unaltered during the first week in all the culture conditions, and therefore pooled data for days 3 and 7 from all morphologically 'normal' chondrocytes following culture under various conditions are shown in Fig. 3.

At day 3, chondrocyte volume increased significantly under the influence of different culture media. At day 0 , chondrocyte volume at the injury was $711 \pm 25 \mu \mathrm{m}^{3}$ and it remained constant by day 3 when explants were cultured in serum-free DMEM (Fig. 3A(a)). Chondrocyte volume at the injury in SF-DMEM or FCS-DMEM culture increased $\sim 55 \%$ at day 3 compared to serum-free DMEM and $\sim 70 \%$ compared to day 0 (Fig. $3 \mathbf{A}(\mathbf{a}) ; p<0.0001$ for both time points) and was greater with SF-DMEM and FCS-DMEM compared to that distant from the injury (Fig. 


\section{Chondrocyte volume}

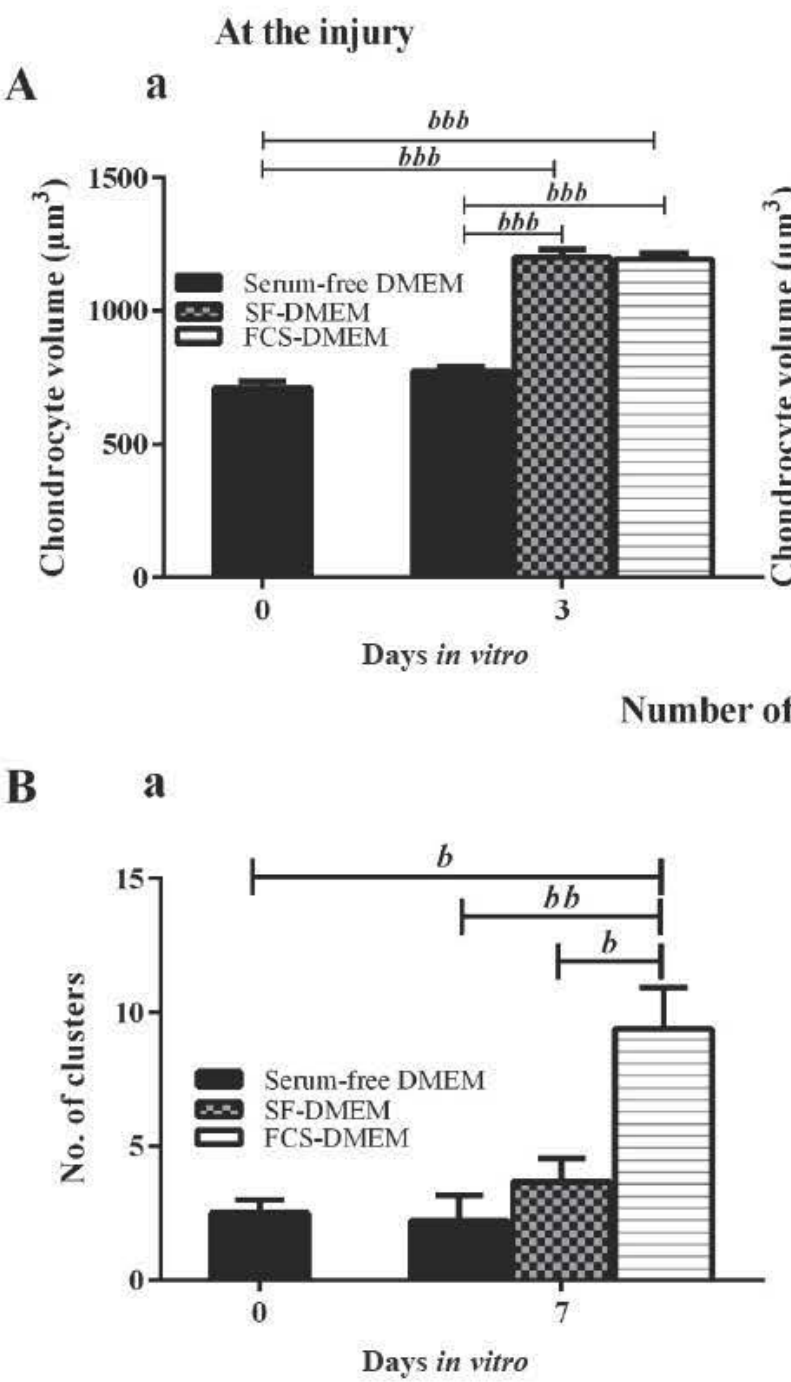

\section{Distant from the injury}

b

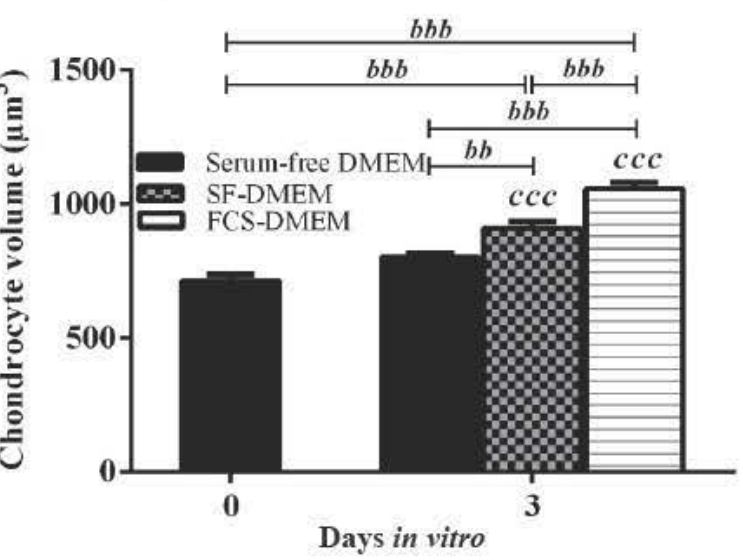

clusters
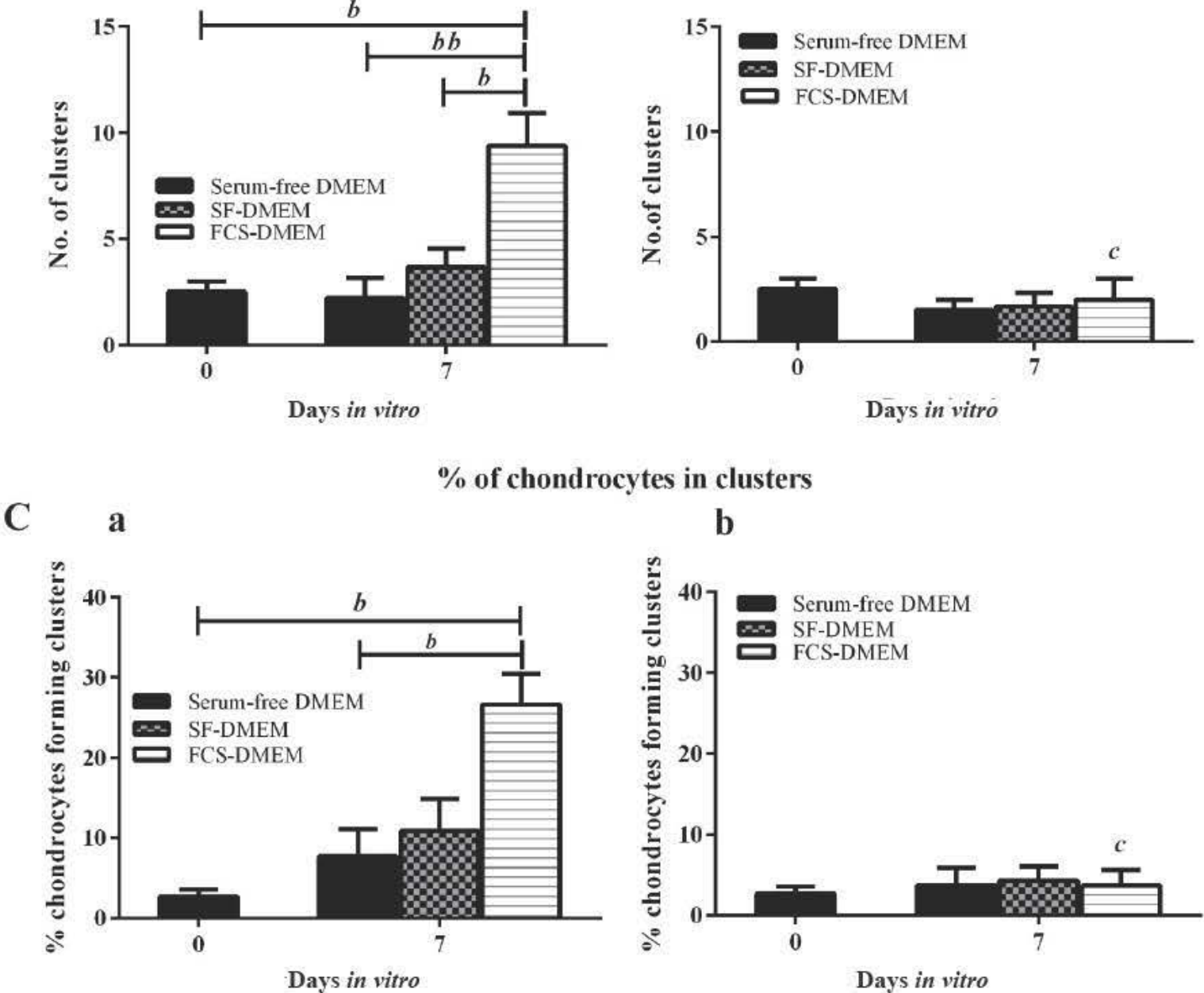

b 


\section{Injured osteochondral explants (full-depth)}

A
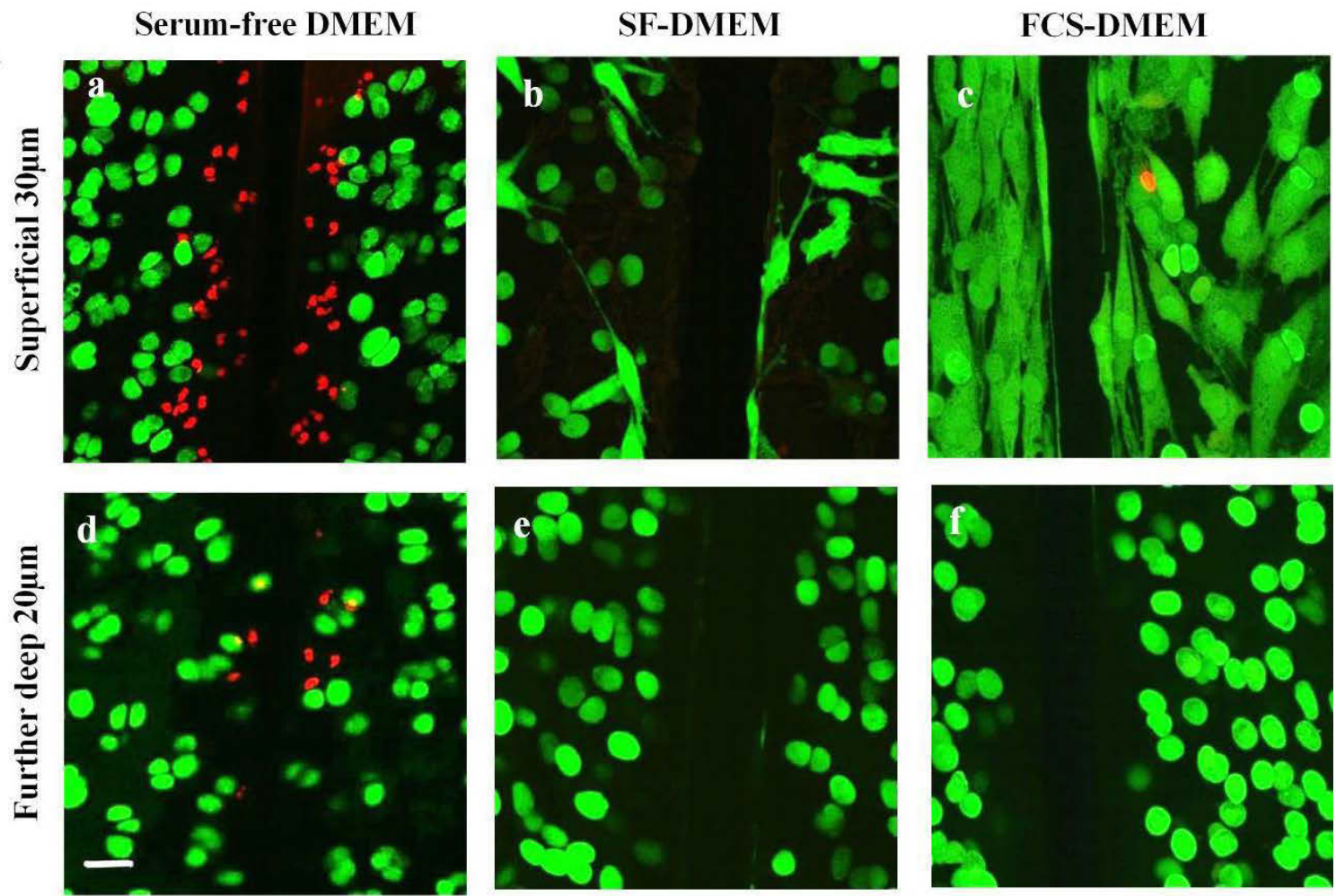

B

Injured osteochondral explants (without superficial layers)
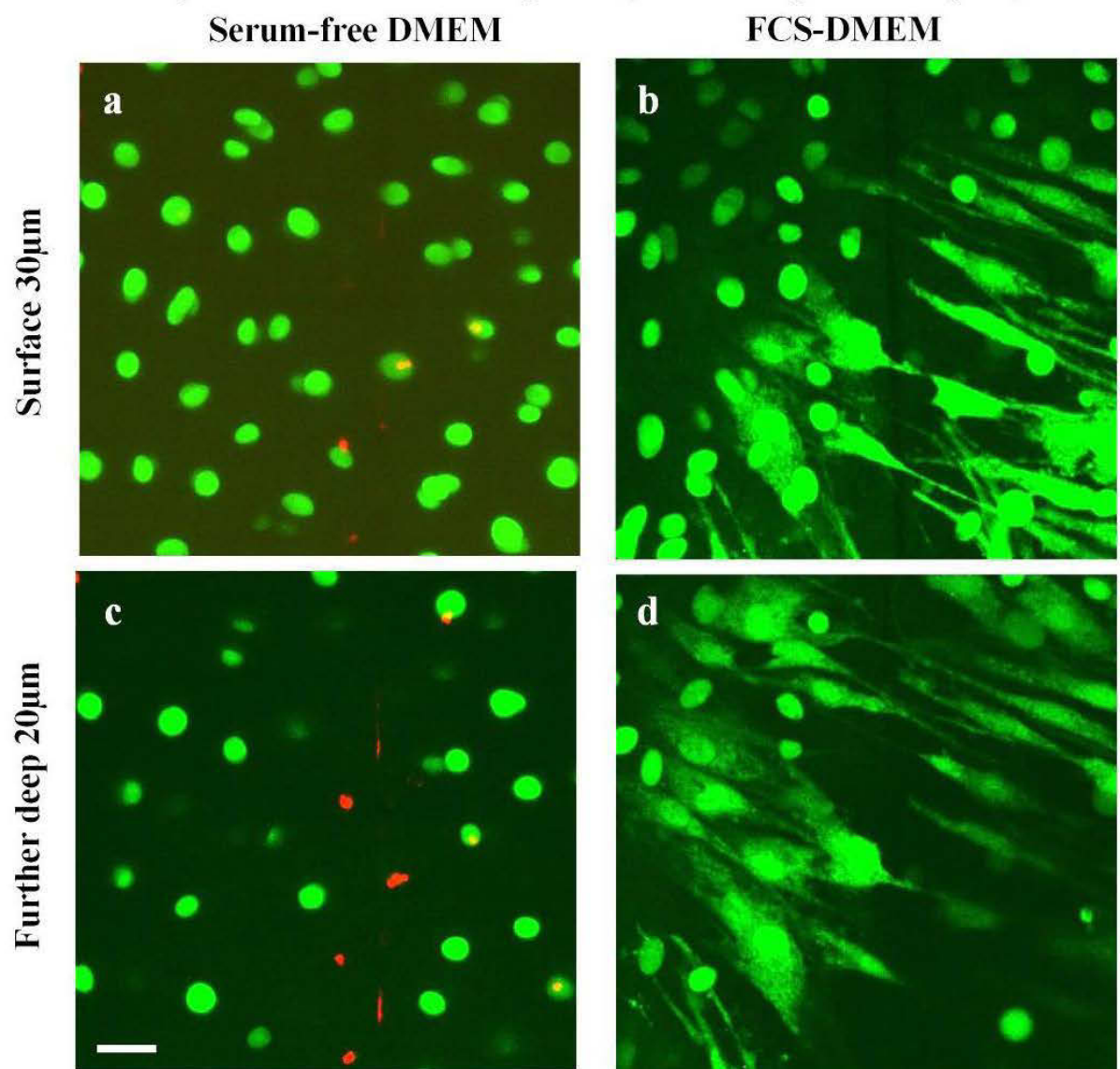

Fig. 4. Heterogeneity of chondrocyte morphology by day 14 at the injury in response to different culture media. Axial CLSM reconstructions ( $\times 40 \mathrm{DW}$ ) of fluorescently-labelled chondrocytes at day $14,(\mathbf{A}(\mathbf{a}, \mathbf{b}, \mathbf{c}))$ superficial $30 \mu \mathrm{m}$ and $(\mathbf{A}(\mathbf{d}, \mathbf{e}, \mathbf{f}))$ a further deep $20 \mu \mathrm{m}$ of the full depth cartilage explants cultured in serum-free DMEM, SF-DMEM and FCS-DMEM, respectively, and $(\mathbf{B}(\mathbf{a}, \mathbf{b}))$ surface $30 \mu \mathrm{m}$ and $(\mathbf{B}(\mathbf{c}, \mathbf{d}))$ a further deep $20 \mu \mathrm{m}$ of the cartilage explants without superficial layers cultured in serum-free DMEM or FCS-DMEM, respectively. Scale bar for all panels $=25 \mu \mathrm{m}$. 
$3 \mathbf{A}(\mathbf{b}) ; p<0.0001$ and $p=0.0002$ for both conditions). However, in serum-free DMEM, no difference in volume was observed at or distant from the injury (Fig. $3 \mathbf{A}(\mathbf{b})$ ).

Chondrocyte clustering was a feature routinely observed at the injury with SF-DMEM or FCS-DMEM culture, but only rarely with serum-free DMEM. At day 0 in normal osteochondral explants, chondrocytes were typically present in pairs (Sasazaki et al., 2008) and only approximately $3 \%$ of chondrocytes within the region of interest (ROI) formed approximately 3-4 clusters with no more than 3 cells per cluster. However, the number of clusters formed in the presence of FCS-DMEM was significantly higher compared to day $0(p<0.05)$ and serum-free DMEM at day $7(p<0.01)$ (Fig. 3B(a)), and also those present in SF-DMEM culture $(p<0.05$; Fig. $3 \mathbf{B}(\mathbf{a}))$. This suggested a more potent action of FCS on cluster formation at the site of injury compared to SF. Distant from the injury, the number of clusters formed was very low under all conditions (Fig. 3B(b)) and significantly less for FCS-DMEM compared to those present at the injury $(p=0.03)$.

In explants cultured with FCS-DMEM, a significantly higher percentage of chondrocytes formed clusters $(27 \pm 4 \%)$ compared to those in serum-free DMEM $(7 \pm 3 \% ; p<0.05)$ and the percentage of chondrocytes forming clusters at day 0 (Fig. $3 \mathbf{C}(\mathbf{a}) ; 3 \pm 1 \%, p<0.05$ ). However, in the presence of SF-DMEM, $11 \pm 4 \%$ chondrocytes formed clusters but the effect was rather weak, and compared to serum-free DMEM, was not statistically significant. Despite this, in explants cultured with FCS-DMEM, a significantly higher percentage of chondrocytes formed clusters at, compared to distant, from the injury (Fig. $3 \mathbf{C}(\mathbf{b}) ; p=0.01$ ) again suggesting a more potent action of FCS-DMEM compared to SF-DMEM. Clusters of chondrocytes were only rarely observed distant from the injury (Fig. 3C(b)). These results suggested that chondrocyte clustering was triggered at the injury in the presence of SF-DMEM and FCS-DMEM compared to serum-free DMEM, and FCS appeared to be more potent compared to SF.

\section{Chondrocyte morphology in response to various culture media in superficial and deeper layers following cartilage injury}

In injured osteochondral explants, the morphology of chondrocytes cultured in serum-free DMEM was normal after $14 \mathrm{~d}$, both in the superficial and deeper regions of cartilage (Fig. 4A(a,d)). In the presence of SF-DMEM or FCS-DMEM, chondrocytes showed marked abnormal morphology, which was restricted to the superficial zones (approx. $30 \mu \mathrm{m}$ depth) of cartilage (Fig. 4A(b,c)). However, the chondrocytes residing deeper than this demonstrated relatively normal volume/morphology (Fig. 4A(e,f)). Similarly, when cartilage explants without subchondral bone were injured, only chondrocytes in the superficial layers at the injury demonstrated abnormal morphology in the presence of SF-DMEM or FCS-DMEM, whereas the morphology of chondrocytes at the injury following culture in serum-free DMEM was unaltered (data not shown). This suggested that the heterogeneous population of cells evident at the injury were morphologically-abnormal chondrocytes induced by SF or FCS, and that cells or other factors from bone were not involved.

In further experiments, the superficial zone from osteochondral explants was surgically removed, and then the injury performed and the samples cultured (Fig. 4B). The chondrocytes still displayed marked morphological changes in the presence of FCS-DMEM compared to serum-free DMEM where the morphology remained relatively normal (Fig. 4B(a,c) versus (b,d)). Thus, FCSDMEM altered the morphology of chondrocytes within the deeper cartilage regions in a similar way to those in the superficial zones. This suggested that FCS-DMEM could induce abnormal morphology to chondrocytes deeper into cartilage, and that these morphological changes were not an exclusive property of chondrocytes in the superficial regions of cartilage.

\section{Injury width and chondrocyte properties following culture in normal or hyperosmotic media with or without FCS}

The effects of FCS on chondrocyte morphology at the injury (volume, clusters, abnormal morphology) were largely overcome by raising the osmolarity of the culture medium. The measurements of morphological characteristics of chondrocytes (volume, abnormal chondrocytes and length of cell bodies) were performed at day 14. At the start of the experiment (day 0), the width of the injury was significantly less in hyperosmolar serum-free DMEM compared to serum-free DMEM (Fig. $5 \mathbf{A}(\mathbf{a}, \mathbf{b})$ and Fig. $5 \mathbf{B} ; p<0.001$ ). By day 14 , injury width had increased significantly in serum-free DMEM, but was unchanged in hyperosmolar serum-free DMEM, whereas the width was significantly less in the presence of FCS-DMEM because of the presence of abnormally-shaped chondrocytes (Fig. $5 \mathbf{A}(\mathrm{m})$ and Fig. $5 \mathbf{B} ; p<0.001)$. Abnormal chondrocyte morphology was evident in FCS-DMEM (Fig. 5A(m)) but not in hyperosmolar FCS-DMEM (Fig. 5A(n)), serum-free DMEM or hyperosmolar serum-free DMEM (Fig. 5A(k,l)), as under these three conditions chondrocyte morphology remained relatively normal (i.e. spheroidal).

Chondrocyte volume following culture in serum-free DMEM, was unchanged after $14 \mathrm{~d}$, but was significantly increased in FCS-DMEM (Fig. 6A(a); $p<0.0001$ ). In hyperosmolar serum-free DMEM, chondrocytes were initially shrunken by approximately $24 \%$ (Fig. 6A(b); $p<0.001$ ) but their volume recovered by day 14 to a level not significantly different from that present in serum-free DMEM. At day 14, cell volume was significantly greater in FCS-DMEM compared to the serum-free DMEM control, but was markedly reduced $(p<0.001)$ in hyperosmolar FCS-DMEM (Fig. 6A(b)). Chondrocytes with cytoplasmic processes were rarely observed following culture in serum-free DMEM (Fig. 6B(a)). However, their presence was markedly stimulated by culture with FCS-DMEM $(p<0.001)$ and completely abolished in hyperosmolar FCS-DMEM (Fig. 5A(n) and Fig. 6B(b)). The normal length of the chondrocyte body in serum-free DMEM was approximately $15 \mu \mathrm{m}$, and this increased by approximately 4-fold in the presence of FCS-DMEM to approximately $56 \mu \mathrm{m}$ (Fig. 6C(a); $p<0.001$ ). In hyperosmolar FCSDMEM, however, the lengths of the cell bodies were 
A

Serum-free DMEM $\quad \begin{aligned} & \text { Hyperosmolar } \\ & \text { serum-free DMEM }\end{aligned} \quad$ FCS-DMEM $\quad \begin{aligned} & \text { Hyperosmolar } \\ & \text { FCS-DMEM }\end{aligned}$
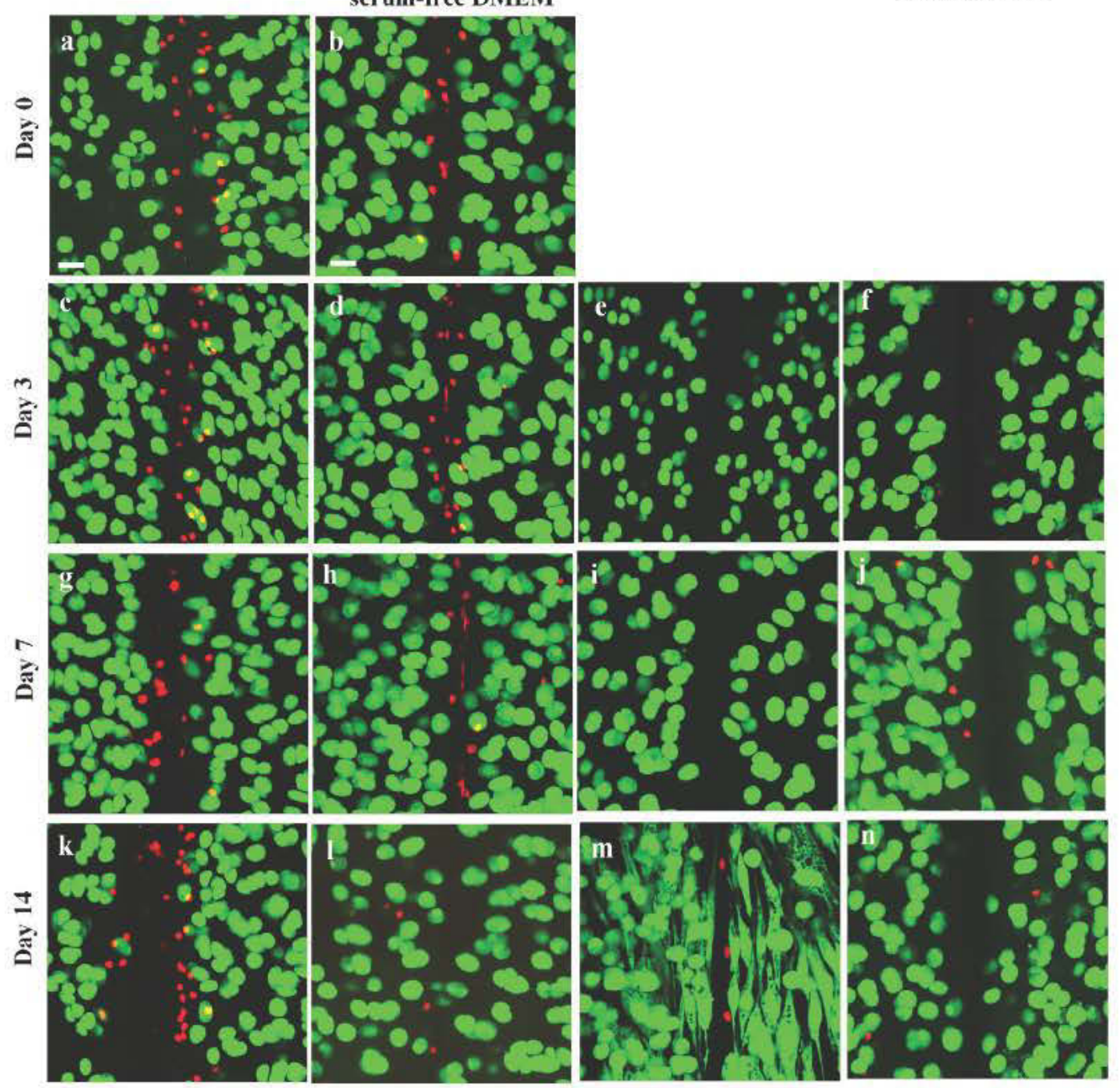

B

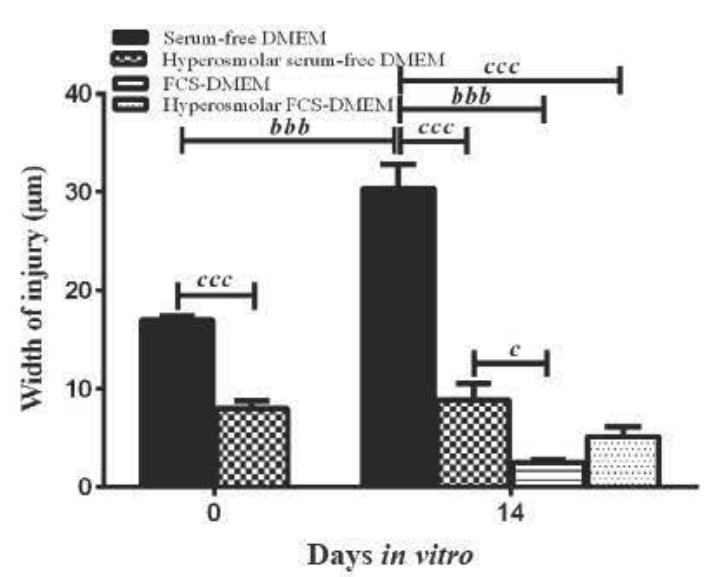

Fig. 5. Injured cartilage explants cultured in serum-free DMEM, hyperosmolar serum-free DMEM, FCS-DMEM and hyperosmolar FCS-DMEM. (A) CLSM reconstructions of high power ( $\times 40 \mathrm{DW})$ magnification images of CMFDA/ PI-labelled chondrocytes (live/dead cells, respectively). The two left panels contain images (a, b) at day $0,(\mathbf{c}, \mathbf{d})$ at day $3,(\mathbf{g}, \mathbf{h})$ at day 7 and $(\mathbf{k}, \mathbf{l})$ at day 14 of the explants exposed to two osmolarities and cultured in the presence of serumfree DMEM and hyperosmolar serum-free DMEM, respectively. The two right panels contain images $(\mathbf{e}, \mathbf{f})$ at day 3 , (i, $\mathbf{j})$ at day 7 and $(\mathbf{m}, \mathbf{n})$ at day 14 of explants exposed to two osmolarities and cultured in the presence of FCS-DMEM and hyperosmolar FCS-DMEM, respectively. Scale bar for all panels $=25 \mu \mathrm{m}$. (B) Width of the injury in explants cultured in the presence of standard and hyperosmolar culture media. $b$ indicates a significant difference between culture media of similar osmolarity at two time points, and $c$ indicates a significant difference between normal osmolarity and hyperosmolar culture media. Single, double and triple symbols showed the level of significance for $p<0.05,0.01$ and 0.001 , respectively. Data were from $[N(n)=8(21)]$. 

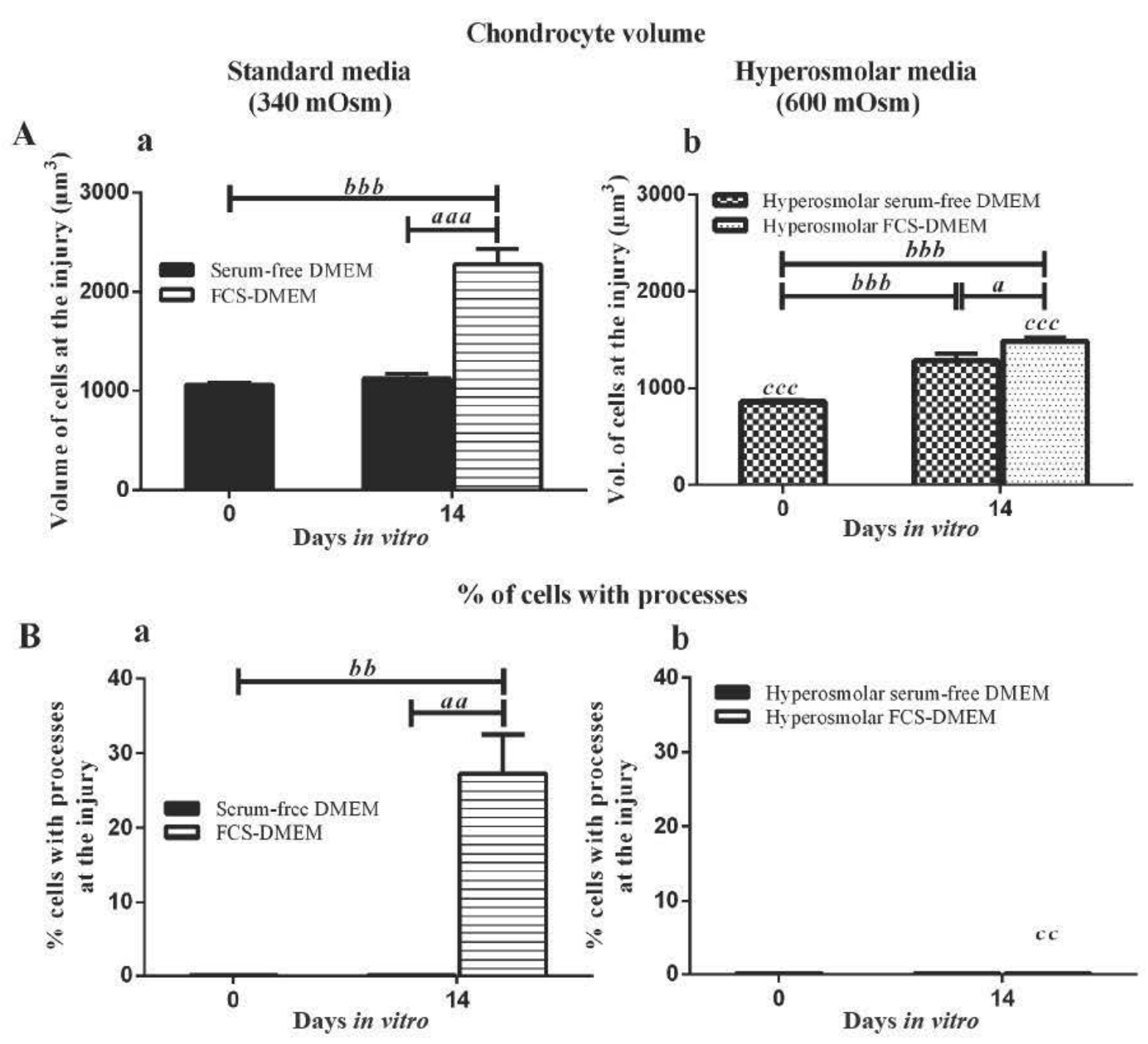

Length of cell body
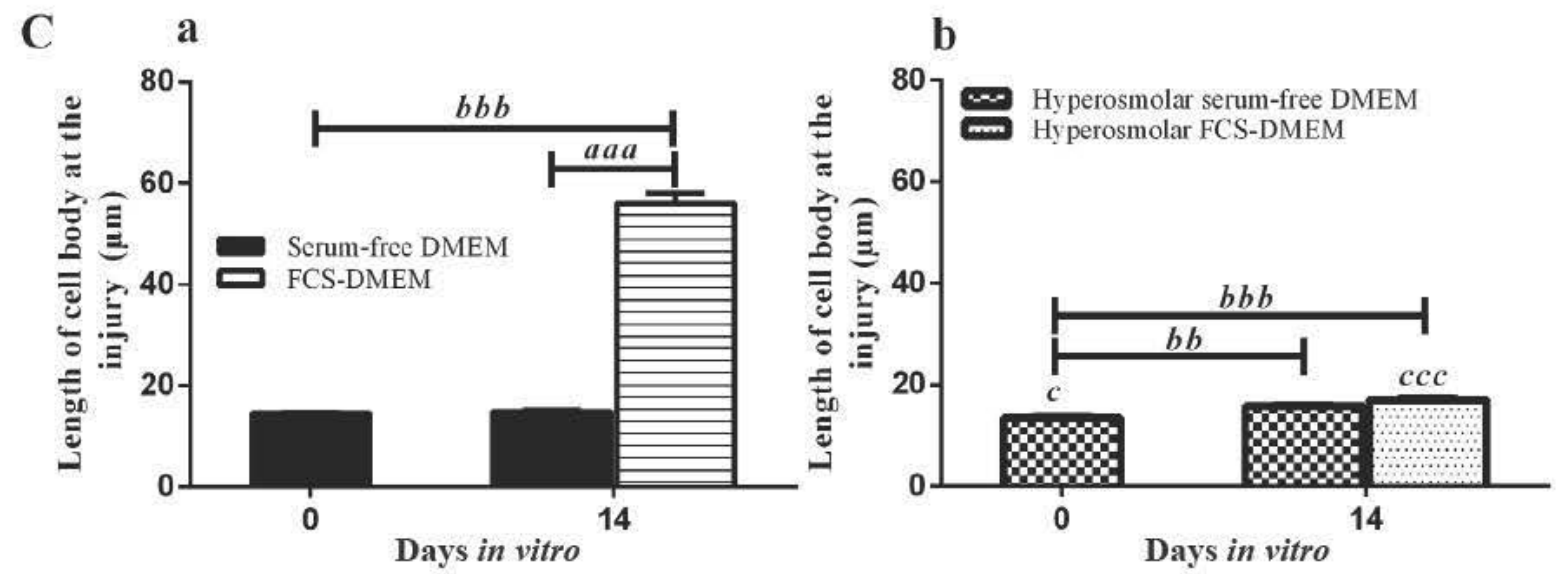

Fig. 6. The heterogeneous nature of chondrocyte morphology at the injury following exposure to serum-containing/ serum-free DMEM (340 mOsm) or hyperosmolar DMEM (600 mOsm) culture media by day 14. Graphs in the left panels (A(a), B(a), C(a)) display pooled data in the presence of serum-free DMEM or FCS-DMEM for cell volume $\left(\mu \mathrm{m}^{3}\right), \%$ of abnormal cells and length of cell bodies $(\mu \mathrm{m})$ at the injury, respectively. The right panels contain graphs $(\mathbf{A}(\mathbf{b}), \mathbf{B}(\mathbf{b}), \mathbf{C}(\mathbf{b}))$ which show pooled data in the presence of hyperosmolar serum-free DMEM or hyperosmolar FCSDMEM for cell volume $\left(\mu \mathrm{m}^{3}\right), \%$ of abnormal cells and average length of cell bodies $(\mu \mathrm{m})$ at the injury, respectively. $a$ indicates a significant difference between two culture media of similar osmolarity, $b$ indicates a significant difference between culture media at two time points, and $c$ indicates a significant difference between normal osmolarity and hyperosmolar culture media. Single, double and triple symbols showed the level of significance for $p<0.05,0.01$ and 0.001 , respectively. Data were from $[N(n)=8(24)]$. 
markedly reduced $(p<0.001)$ to levels close to those observed in hyperosmolar serum-free DMEM $(17 \pm 0.4 \mu \mathrm{m}$ and $16 \pm 0.4 \mu \mathrm{m}$, respectively; Fig. $6 \mathbf{C}(\mathbf{b}))$.

For measurements of chondrocyte clusters, a shorter incubation period of $7 \mathrm{~d}$ was required because after this chondrocytes were too tightly packed making discrimination of the membrane and thus identification of the cells inaccurate. In the presence of FCS-DMEM, the number of clusters and percentage of chondrocytes forming clusters at the injury increased significantly compared to serum-free DMEM ( $p=0.02$ and $p=0.01$, respectively) and also in comparison to day 0 (Fig. 7A(a) and $7 \mathbf{B}(\mathbf{a}) ; p<0.01$ and $p<0.001)$. By day 7 , the average number of clusters and percentage of chondrocytes forming clusters remained significantly less in the presence of hyperosmolar serum-free DMEM and hyperosmolar FCS-DMEM compared to serum-free DMEM and normal osmolarity FCS-DMEM, respectively ( $p<0.05$ for both the conditions; Fig. 7A(b) and $7 \mathbf{B}(\mathbf{b})$ ). Taking these results together, FCS-DMEM increased chondrocyte volume, the $\%$ of cells with processes, the length of the cell body, the number of clusters and the $\%$ of chondrocytes within clusters. However, all these parameters were markedly suppressed $(0.01<p<0.001)$ if the osmolarity of the FCS-DMEM was raised to $600 \mathrm{mOsm}$.

\section{Discussion}

Partial-thickness cartilage injury initiated substantial changes to in situ chondrocytes, particularly following culture with synovial fluid (SF-DMEM) or foetal calf serum (FCS-DMEM). At the site of scalpel injury during culture in serum-free DMEM, despite an increase in injury width, there were no other significant changes to chondrocyte properties. In marked contrast, when injured explants were cultured with SF-DMEM or FCS-DMEM there were increases in cell volume, development of morphologicallyabnormal cells with extended cytoplasmic processes, and cell clustering. Since relatively minor changes to chondrocytes distant from the injury were observed in SF-DMEM or FCS-DMEM culture, this suggested that penetration of factors at the site of injury could account for the development of these abnormal chondrocyte properties. However, the morphological changes to chondrocytes observed at the injury during culture with FCS-DMEM, were largely abolished when medium osmolarity was increased (hyperosmolar FCS-DMEM). These results raise a number of key questions about the response of chondrocytes following partial-thickness injury.

Culture medium composition strongly influenced the properties of injured cartilage and chondrocytes. In serum-free DMEM, injury width increased over $14 \mathrm{~d}$ (Fig. 1B), probably due to collagen damage, leading to further water imbibition by matrix proteoglycans (PGs) (Maroudas, 1976) resulting in tissue swelling at the site of injury. This coupled with the progressive loss of glycosaminoglycans (GAGs) during culture (Torzilli and Grigiene, 1998), would decrease extracellular osmolarity and stimulate chondrocyte swelling (Bush and Hall, 2001a). However, chondrocyte volume at the site of injury did not change during culture (Fig. 2C(a)) suggesting that volume regulation had occurred (Bush and Hall, 2001b). For explants cultured in SF-DMEM or FCS-DMEM, injury width also increased, but between 7 and $14 \mathrm{~d}$ it decreased (Fig. 1B) because of the presence of chondrocytes with abnormal morphology (Fig. 1A(g,j)). This was probably due to the enhanced permeability of the damaged cartilage (Armstrong and Mow, 1982; Byun et al., 2013) allowing factors present in SF or FCS access to the chondrocytes at and near the injury. In contrast, chondrocyte morphology distant from the injury was relatively normal (Fig. 2B(b) and Fig. $2 \mathbf{D}(\mathbf{b})$ ), supporting the notion that the undamaged matrix retained its relative impermeability to morphogenic factors. As a first step towards identifying these factors, preliminary experiments were performed with heatinactivated FCS (HI-FCS; $56{ }^{\circ} \mathrm{C}, 30 \mathrm{~min}$ ). The results indicated no difference in the changes occurring at the injury site with chondrocytes with abnormal morphology being observed following culture in both conditions after $14 \mathrm{~d}$ (Karim, A. unpublished results). This temperature treatment destroys the complement system (Triglia and Linscott, 1980) indicating that these elements are probably not involved in mediating the morphological changes. Future experiments using other procedures to eliminate other possible ingredients in serum, would be of particular interest in order to identify the active agent(s) responsible for inducing the morphological changes.

There was a significant loss of dead (PI-labelled) chondrocytes following culture in the presence of SFDMEM and FCS-DMEM compared to serum-free DMEM (Fig. 1A,C). This was probably due to the presence of nucleic acid-degrading enzymes (Zhou et al., 2011). As the fluorescent dye (PI) was added $1 \mathrm{~h}$ prior to imaging, this suggests little additional cell death after injury. This was probably because of the focal nature of the injury, as relatively non-specific damage (e.g. impact injury) may result in progressive cell death (Redman et al., 2004). Notably, at day 3 , the effect of FCS on PI-labelled chondrocytes was greater than SF (Fig. 1C), providing additional cautionary evidence (e.g. Martin et al., 2002) that FCS-supplemented culture media may have a more potent effect on chondrocyte and cartilage properties compared to SF.

By day 14, chondrocyte properties at the site of injury were markedly altered following culture with SFDMEM or FCS-DMEM (Fig. 2A-D). The orientation of the cytoplasmic processes in FCS-DMEM was similar to that previously reported (Lyman et al., 2012); however, their presence in SF-DMEM and absence in serum-free DMEM (Fig. 1A) have not yet been described. Distant from the injury, these effects were markedly reduced but still present, and significantly greater than those observed during culture with serum-free DMEM. The volume of chondrocytes with normal morphology near the injury increased in the presence of SF or FCS compared to serum-free DMEM (Fig. 3A(a)). The initial volume of chondrocytes $\left(711 \pm 25 \mu \mathrm{m}^{3}\right)$ was within the range of superficial zone cells in intact (uninjured) bovine articular cartilage (Bush and Hall, 2001a). By day 3, there was a $55 \%$ increase in the chondrocyte volume near the injury in SF-DMEM or FCS-DMEM compared to serum-free 


\section{Number of clusters}

\section{Standard media (340 mOsm)}

A

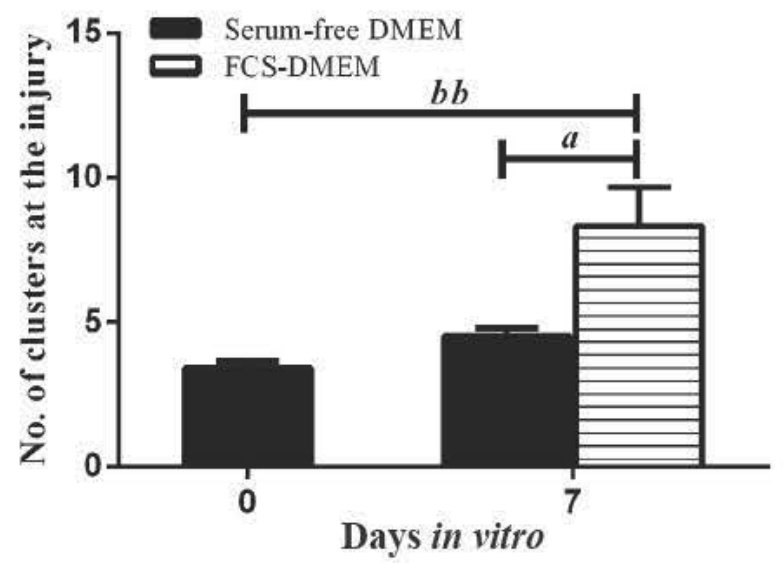

\section{Hyperosmolar media (600 mOsm)}

b

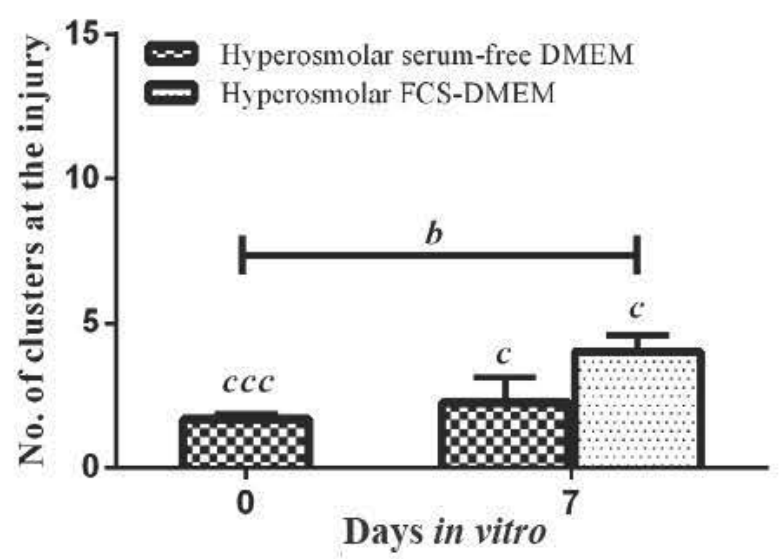

$\%$ of chondrocytes in clusters

B

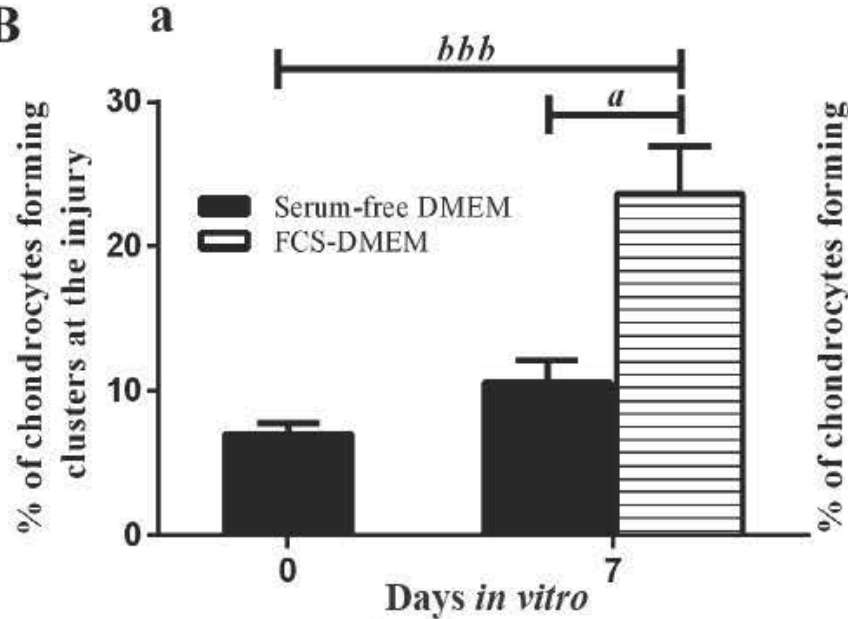

b

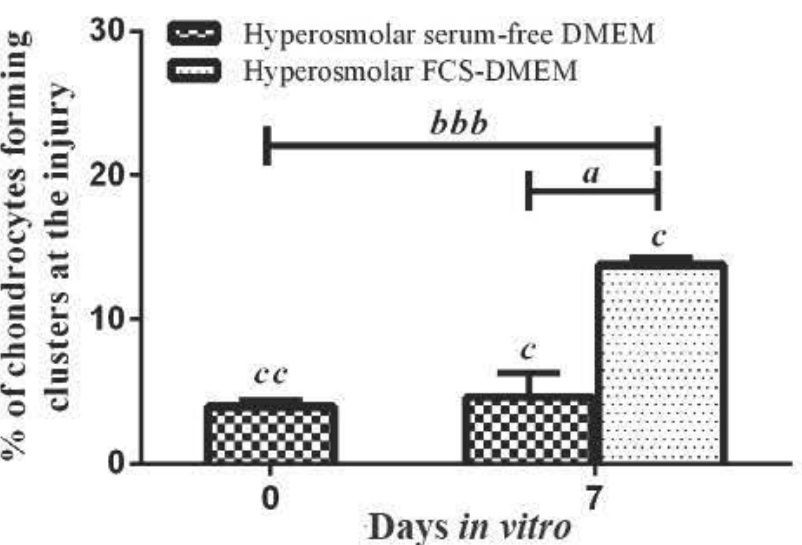

Fig. 7. Clusters formed at the injury in the injured explants after exposure to serum-containing/serum-free DMEM (340 mOsm) or hyperosmolar DMEM (600 mOsm) culture media. By day 7 (A, B) average number of clusters and $\%$ of chondrocytes forming clusters at the injury in (a) standard and (b) hyperosmolar culture media after exposure to $340 \mathrm{mOsm}$ or $600 \mathrm{mOsm}$, respectively. $a$ indicates a significant difference between two culture media of similar osmolarity, $b$ indicates a significant difference between culture media at two time points, and $c$ indicates a significant difference between normal osmolarity and hyperosmolar culture media. Single, double and triple symbols showed the level of significance for $p<0.05,0.01$ and 0.001 , respectively. Data were from $[N(n)=8(26)]$.

DMEM (Fig. 3A(a)) and those distant (32\% in SF and $13 \%$ in FCS) from the injury (Fig. $3 \mathbf{A}(\mathbf{b}))$. These findings are similar to the chondrocyte swelling (Bush and Hall, 2003) and hypertrophic-like changes observed in degenerative osteoarthritic (OA) cartilage (Van der Krann and van den Berg, 2012). It would be of interest to follow the changes to chondrocyte morphology in more detail to ascertain if chondrocyte swelling occurred before the development of cytoplasmic processes.

Chondrocyte clustering was another key morphological feature observed in our model (Fig. 3) and is also a hallmark of OA (Mankin et al., 1971; Lotz et al., 2010). In fresh non-degenerate bovine cartilage, chondrocytes typically occur in pairs (Sasazaki et al., 2008) with very few cells in clusters ( $\geq 3$ cells; Fig. 3B,C). After 7 d, a few clusters formed at the injury in serum-free DMEM, however under the effect of SF or FCS, marked clustering was evident (Fig. $3 \mathbf{B}(\mathbf{a})$ and $\mathbf{C}(\mathbf{a})$ ), suggesting the action of growth factors (Martin et al., 2002; Khan et al., 2010). The action of FCS on the number and \% of chondrocytes forming clusters was more potent than SF (Fig. 3B(a) and C(a)) (Martin et al., 2002). In contrast, chondrocytes distant from the 
injury exhibited almost no cluster formation (Fig. $3 \mathbf{B}(\mathbf{b})$ and $\mathbf{C}(\mathbf{b})$ ), probably because the matrix was relatively intact thereby hindering penetration of cluster-stimulating factors.

Chondrocytes with abnormal morphology were observed in superficial regions of injured explants cultured in SF-DMEM or FCS-DMEM but not in serum-free DMEM (Fig. 1A(d,g,j) and 2B). The imaging method visualised cells to a depth of approximately $30 \mu \mathrm{m}$; however, imaging a further $20 \mu \mathrm{m}$ deeper into this region demonstrated that chondrocyte morphology was spheroidal and relatively 'normal' (Fig. 4A(e,f)). This raised the question of whether only chondrocytes within the superficial regions were sensitive to the effects of SF or FCS. However, when the superficial layer of cartilage was removed, following injury the cells in deeper layers also changed shape (Fig. $4 \mathbf{B}(\mathbf{b}, \mathbf{d})$ ) in the presence of FCS, and thus the ability to alter morphology did not appear to be a unique feature of chondrocytes in the superficial regions of injured cartilage.

In hyperosmotic serum-free DMEM at day 0, injury width was less compared to serum-free DMEM resulting in reduced chondrocyte death (Fig. 5B). This might appear to conflict with a recently-published in vivo study where injury width was not significantly different between media of normal or raised osmotic pressure (Eltawil et al., 2015). However, the cartilage injury was previously performed on the whole joint whereas in the current work osteochondral explants were used. Thus, in the latter model, cartilage shrinking may have occurred leading to reduced injury width. Interestingly, the injury width following culture in hyperosmolar serum-free DMEM did not change over $14 \mathrm{~d}$ whereas it increased by more than 2-fold in serum-free DMEM (Fig. 5B). A 'protective' effect of raised osmolarity has already been described following injury to cartilage subjected to impact (Bush et al., 2005), and scalpel injury both in vitro (Amin et al., 2008) and in vivo (Eltawil et al., 2015).

Injury width decreased in FCS-DMEM by day 14 due to the development of abnormal chondrocytes (Fig. 5A(m)); however, no abnormal cells were observed in hyperosmolar FCS-DMEM (Fig. 5A(n)). Several properties of chondrocytes previously shown to be altered in SF-DMEM or FCS-DMEM (Fig. 2) were significantly suppressed in hyperosmolar serum-free DMEM or hyperosmolar FCS-DMEM (Fig. 6). Chondrocyte volume was reduced (Fig. 6A(b)), and there were almost no cells with cytoplasmic processes or with an increased cell body length compared to serum-free DMEM or FCS-DMEM (Fig. 6B and C). Thus, hyperosmolarity almost completely inhibited the chondrocyte volume and morphological changes observed with FCS-DMEM and the injured cartilage had the appearance of that in serum-free DMEM (Fig. 1A(d) compared to Fig. 5A(n)). [Note however that at day 14, PI-labelled chondrocytes were still observed in serum-free DMEM, whereas they had disappeared in hyperosmolar FCS-DMEM]. These results suggest a close association between the changes to chondrocyte morphology induced by FCS and their inhibition by increased culture medium osmolarity.
Chondrocyte phenotype and expression of many genes encoding for cartilage extracellular matrix proteins are known to be strongly regulated by the SOX 9 transcription factor (de Crombrugghe et al., 2000; Tew et al., 2005). This in turn is controlled by medium osmolarity (Tew et al., 2009) which is known to regulate the p38 mitogenactivated protein kinase (MAPK) pathway in many organisms (Sheikh-Hamad and Gustin, 2004). One possible explanation for these results is that the raised osmolarity increases chondrocyte SOX9 mRNA stability and SOX9 protein production (Tew et al., 2009) and overcomes the actions of SF-DMEM or FCS-DMEM which stimulate abnormal chondrocyte morphology, resulting in normal (spheroidal) cell shape. The results also demonstrated that increasing osmolarity reduced chondrocyte cluster formation and the $\%$ of chondrocytes in clusters, despite the presence of FCS (Fig. 7A and B). Thus, although there would appear to be deleterious effects on chondrocytes, these may be largely overcome by raising osmolarity. It is established that the mechanical properties of 'repair' tissue following injury are poor and may possess fibrocartilaginous characteristics (Hunziker, 1999; Hunziker, 2002). The present results show that factors in SF or FCS may stimulate morphological and potentially phenotypic changes to chondrocytes which might not then produce mechanically-resilient cartilaginous-like tissue. However, raising osmolarity would appear to protect chondrocytes from these deleterious effects and perhaps in combination with growth factors (Hunziker, 2001) could stimulate more effective cartilage repair. Recent studies using a partial-thickness in vivo model showed that the repair score, type II collagen, aggrecan levels and injury width were significantly improved when injury was applied in the presence of elevated (600 mOsm) compared to 'normal' osmolarity (Eltawil et al., 2015).

A key question was whether the abnormal cells observed at the injury were existing chondrocytes undergoing changes to morphology, or whether they were chondroprogenitor cells (CPCs) present within the superficial zone (Alsalameh et al., 2004; Dowthwaite et al., 2004) which had migrated to the injury. Recent work has suggested that CPCs may be attracted by chemotactic factors (collectively known as 'alarmins') from dead (necrotic) chondrocytes or in enzymaticallytreated cartilage, and stimulated to repopulate the injured site (Seol et al., 2012; Seol et al., 2014). While we do not believe our data allow a firm conclusion, there are some observations that are relevant. No morphologicallyabnormal cells were observed within injured cartilage cultured in serum-free DMEM (Fig. 1A(d)) despite the presence of dead (PI-labelled) chondrocytes. However, this could be because levels of 'alarmins' might be too low and the scalpel injury highly focused compared to wounding by blunt impact or scratching (Seol et al., 2012). In contrast, many abnormal cells were observed at the injury in SF-DMEM or FCS-DMEM despite the fact that dead cell material was removed relatively rapidly (e.g. day 3; Fig. 1A(e,h)). While chondrocytes with abnormal morphology were rarely observed distant from the injury (Fig. $2 \mathbf{B}(\mathbf{b})$ ), there was no suggestion that they were 
migrating in a preferential direction towards the injury (Karim, A. unpublished results). In addition, changes to chondrocyte shape were observed when the superficial layer was substantially removed suggesting that these changes were not exclusive to cells in this region (Fig. 4B). In hyperosmolar FCS-DMEM, cells with processes were rarely observed (Fig. $6 \mathbf{B}(\mathbf{b})$ ), which could be taken to indicate suppression of CPCs; however, it has been reported that raising medium osmolarity significantly increased chondrogenic gene expression during the course of chondrogenic differentiation of progenitor cells (Caron et al., 2013). There is no doubt, however, that both SF and FCS are key determinants of the altered cell morphology, and may stimulate existing chondrocytes at the site of injury, or act as a co-factor in the recruitment/migration of CPCs to the repair site.

We have utilised CLSM and quantitative imaging to determine properties of in situ chondrocytes at and distant from a partial-thickness scalpel injury applied to a bovine osteochondral explant model. Culture in SF-DMEM or FCS-DMEM produced marked changes to properties of chondrocytes at the injury, stimulating increased cell volume, development of abnormal morphology and clustering, with minor effects on cells distant from the injury. The finding that increased osmolarity markedly suppressed the FCS-induced changes to chondrocytes suggested a common site of action and served to preserve normal chondrocyte morphology.

\section{Acknowledgements}

AK thanks the College of Medicine and Veterinary Medicine, University of Edinburgh, for a Global Overseas Research Scholarship and Charles Darwin Scholarship, and the University of Health Sciences, Lahore and Higher Education Commission, Pakistan for support. We also thank Dr. Trudi Gillespie, IMPACT Facility, Centre for Integrative Physiology, University of Edinburgh, for expert assistance with confocal laser scanning microscopy. We confirm that the authors have no conflicts of interest to declare and there has been no significant financial support for this work that could influence its outcome.

\section{References}

Alsalameh S, Amin R, Gemba T, Lotz M (2004) Identification of mesenchymal progenitor cells in normal and osteoarthritic human articular cartilage. Arthritis Rheum 50: 1522-1532.

Amin AK, Huntley JS, Bush PG, Simpson AHRW, Hall AC (2008) Osmolarity influences chondrocyte death in wounded articular cartilage. J Bone Joint Surg Am 90: 1531-1542.

Amin AK, Huntley JS, Bush PG, Simpson AHRW, Hall AC (2009) Chondrocyte death in mechanically injured articular cartilage - the influence of extracellular calcium. J Orthop Res 27: 778-784.

Amin AK, Huntley JS, Simpson AH, Hall AC (2010) Increasing the osmolarity of joint irrigation solutions may avoid injury to cartilage: a pilot study. Clin Orthop Relat Res 468: 875-884.

Armstrong CG, Mow VC (1982) Variations in the intrinsic mechanical properties of human articular cartilage with age, degeneration, and water content. J Bone Joint Surg Am 64: 88-94.

Bush PG, Hall AC (2001a) The osmotic sensitivity of isolated and in situ bovine articular chondrocytes. J Orthop Res 19: 768-778.

Bush PG, Hall AC (2001b) Regulatory volume decrease (RVD) by isolated and in situ bovine articular chondrocytes. J Cell Physiol 187: 304-314.

Bush PG, Hall AC (2003) The volume and morphology of chondrocytes within non-degenerate and degenerate human articular cartilage. Osteoarthritis Cartilage 11: 242251.

Bush PG, Hodkinson PD, Hamilton GL, Hall AC (2005) Viability and volume of in situ bovine articular chondrocytes - changes following a single impact and effects of medium osmolarity. Osteoarthritis Cartilage 13: 54-65.

Byun S, Sinsky YL, Lu YCS, Ort T, Kavalkovich K, Sivakumar P, Hunziker EB, Frank EH, Grodzinsky AJ (2013) Transport of anti-IL-6 antigen binding fragments into cartilage and the effects of injury. Arch Biochem Biophys 532: 15-22.

Caron MMJ, van der Windt AE, Emans PJ, van Rhijn LW, Jahr H, Welting TJM (2013) Osmolarity determines the in vitro chondrogenic differentiation capacity of progenitor cells via nuclear factor of activated T-cells 5 . Bone 53: 94-102.

Cucchiarini M, Madry H, Ma C, Thurn T, Zurakowski D, Menger MD, Kohn D, Trippel SB, Terwilliger EF (2005) Improved tissue repair in articular cartilage defects in vivo by rAAV-mediated overexpression of human fibroblast growth factor 2. Mol Ther 12: 229-238.

de Crombrugghe B, Lefebvre V, Behringer RR, Bi W, Murakami S, Huang W (2000) Transcriptional mechanisms of chondrocyte differentiation. Matrix Biol 19: 389-394.

Dowthwaite GP, Bishop JC, Redman SN, Khan IM, Rooney P, Evans DJ, Haughton L, Bayram Z, Boyer S, Thomson B, Wolfe MS, Archer CW (2004) The surface of articular cartilage contains a progenitor cell population. J Cell Sci 117: 889-897.

Eltawil NM, Howie SEM, Simpson AHRW, Amin AK, Hall AC (2015) The use of hyperosmotic saline for chondroprotection: implications for orthopaedic surgery and cartilage repair. Osteoarthritis Cartilage 23: 469-477.

Farhan-Alanie MM, Hall AC (2014) Temperature changes and chondrocyte death during drilling in a bovine cartilage model and chondroprotection by modified irrigation solutions. Int Orthop 38: 2407-2412.

Fortier LA, Mohammed HO, Lust G, Nixon AJ (2002) Insulin-like growth factor-I enhances cell-based repair of articular cartilage. J Bone Joint Surg Br 84: 276-288.

Hunziker EB (1999) Articular cartilage repair: are the intrinsic biological constraints undermining this process insuperable? Osteoarthritis Cartilage 7: 15-28.

Hunziker EB (2001) Growth-factor-induced healing of partial-thickness defects in adult articular cartilage. Osteoarthritis Cartilage 9: 22-32. 
Hunziker EB (2002) Articular cartilage repair: basic science and clinical progress. A review of the current status and prospects. Osteoarthritis Cartilage 10: 432-463.

Khan IM, Palmer EA, Archer CW (2010) Fibroblast growth factor-2 induced chondrocyte cluster formation in experimentally wounded articular cartilage is blocked by soluble Jagged-1. Osteoarthritis Cartilage 18: 208-219.

Knutsen G, Engebretsen L, Ludvigsen TC, Drogset JO, Grøntvedt T, Solheim E, Strand T, Roberts S, Isaksen V, Johansen O (2004) Autologous chondrocyte implantation compared with microfracture in the knee. J Bone Joint Surg Am 86: 455-464.

Lotz MK, Otsuki S, Grogan SP, Sah R, Terkeltaub R, D'Lima D (2010) Cartilage cell clusters. Arthritis Rheum 62: 2206-2218.

Lyman JR, Chappell JD, Morales TI, Kelley SS, Lee GM (2012) Response of chondrocytes to local mechanical injury in an ex vivo model. Cartilage 3: 58-69.

Mankin HJ, Dorfman H, Lippiello L, Zarins A (1971) Biochemical and metabolic abnormalities in articular cartilage from osteo-arthritic human hips. II. Correlation of morphology with biochemical and metabolic data. J Bone Joint Surg Am 53: 523-537.

Maroudas A (1976) Balance between swelling pressure and collagen tension in normal and degenerate cartilage. Nature 260: 808-809.

Maroudas A, Venn M (1977) Chemical composition and swelling of normal and osteoarthrotic femoral head cartilage. Ann Rheum Dis 36: 399-406.

Martin JA, Wilkey AL, Brand RA (2002) Cartilage extracellular matrix metabolism differs in serum and synovial fluid. Methods Cell Sci 24: 139-143.

Namba RS, Meuli M, Sullivan KM, Le AX, Adzick NS (1998) Spontaneous repair of superficial defects in articular cartilage in a fetal lamb model. J Bone Joint Surg Am 80: 4-10.

Patwari P, Cook MN, DiMicco MA, Blake SM, James IE, Kumar S, Cole AA, Lark MW, Grodzinsky AJ (2003) Proteoglycan degradation after injurious compression of bovine and human articular cartilage in vitro. Interaction with exogenous cytokines. Arthritis Rheum 48: 1292-1301.

Redman SN, Dowthwaite GP, Thomson BM, Archer CW (2004) The cellular responses of articular cartilage to sharp and blunt trauma. Osteoarthritis Cartilage 12: 106116.

Sasazaki Y, Seedhom BB, Shore R (2008) Morphology of the bovine chondrocyte and of its cytoskeleton in isolation and in situ: are chondrocytes ubiquitously paired through the entire layer of cartilage? Rheumatology 47: 1641-1646.

Seol D, McCabe DJ, Choe H, Zheng H, Yu Y, Jang K, Walter MW, Lehman AD, Ding L, Buckwalter JA,
Martin JA (2012) Chondrogenic progenitor cells respond to cartilage injury. Arthritis Rheum 64: 3626-3637.

Seol D, Yu Y, Choe H, Jang K, Brouillette MJ, Zheng H, Lim TH, Buckwalter JA, Martin JA (2014) Effect of short-term enzymatic treatment on cell migration and cartilage regeneration:in vitro organ culture of bovine articular cartilage. Tissue Eng Part A 20: 1807-1814.

Sheikh-Hamad D, Gustin MC (2004) MAP kinases and the adaptive response to hypertonicity:functional preservation from yeast to mammals. Am J Physiol Renal Physiol 287: F1102-F1110.

Steadman JR, Rodkey WG, Rodrigo JJ (2001) Microfracture: surgical technique and rehabilitation to treat chondral defects. Clin Orthop Relat Res 391S: S362-S369.

Tew SR, Li Y, Pothacharoen P, Tweats LM, Hawkins RE, Hardingham TE (2005) Retroviral transduction with SOX9 enhances re-expression of the chondrocyte phenotype in passaged osteoarthritic human articular chondrocytes. Osteoarthritis Cartilage 13: 80-89.

Tew SR, Peffers MJ, McKay TR, Lowe ET, Khan WS, Hardingham TE, Clegg PD (2009) Hyperosmolarity regulates SOX9 mRNA posttranscriptionally in human articular chondrocytes. Am J Physiol Cell Physiol 297: C898-C906.

Torzilli PA, Grigiene R (1998) Continuous cyclic load reduces proteoglycan release from articular cartilage. Osteoarthritis Cartilage 6: 260-268.

Triglia RP, Linscott WD (1980) Titers of nine complement components, conglutinin and $\mathrm{C} 3 \mathrm{~b}$-inactivator in adult and fetal bovine sera. Mol Immunol 17: 741-748.

Trippel SB (1995) Growth factor actions on articular cartilage. J Rheumatol Suppl 43: 129-132.

Urban JPG, Hall AC, Gehl KA (1993) Regulation of matrix synthesis rates by the ionic and osmotic environment of articular chondrocytes. J Cell Physiol 154: 262-270.

Van der Krann PM, van den Berg WB (2012) Chondrocyte hypertrophy and osteoarthritis: role in initiation and progression of cartilage degeneration? Osteoarthritis Cartilage 20: 223-232.

Wei X, Gao J, Messner K (1997) Maturation-dependent repair of untreated osteochondral defects in the rabbit knee joint. J Biomed Mater Res 34: 63-72.

Zhou S, Cui Z, Urban J (2011) Dead cell counts during serum cultivation are underestimated by the fluorescent live/dead assay. Biotechnol J 6: 513-518.

Editor's Note: All questions/comments by the reviewers were answered by text changes. There is hence no Discussion with Reviewers section. Scientific Editor in charge of the paper: Martin Stoddart. 